\title{
Predicting the Outcome of Photocyclisation Reactions: A Joint Experimental and Computational Investigation
}

\author{
A. D. Dinga Wonanke, Jayne L. Ferguson, Christopher M. Fitchett, and \\ Deborah L. Crittenden ${ }^{*[a]}$
}

Abstract: Photochemical oxidative cyclodehydrogenation reactions are a versatile class of aromatic ring-forming reactions. They are tolerant to functional group substitution and heteroatom inclusion, so can be used to form a diverse range of extended polyaromatic systems by fusing existing ring substituents. However, despite their undoubted synthetic utility, there are no existing models-computational or heuristic - that predict the outcome of photocyclisation reactions across all possible classes of reactants. This can be traced back to the fact that "negative" results are rarely published in the synthetic literature and the lack of a general conceptual framework for understanding how photoexcitation affects reactivity. In this work, we address both of these issues. We present experimental data for a series of aromatically substituted pyrroles and indoles, and show that quantifying induced atomic forces upon photoexcitation provides a powerful predictive model for determining whether a given reactant will photoplanarise and hence proceed to photocyclised product under appropriate reaction conditions. The propensity of a molecule to photoplanarise is related to localised changes in charge distribution around the putative forming ring upon photoexcitation. This is promoted by asymmetry in molecular structures and/or charge distributions, inclusion of heteroatoms and ethylene bridging and well-separated or isolated photocyclisation sites.

\section{Introduction}

Since photochemical reactions were discovered over 100 years ago, photochemical synthesis has become an important branch of organic chemistry. ${ }^{[1,2]}$ Photochemical syntheses involve the absorption of light by a molecule, causing it to transition to an electronically excited state. This results in redistribution of electrons, changing the chemical properties of the molecule and broadening its reactivity spectrum by opening reaction pathways that may not be accessible by conventional thermal procedures. ${ }^{[3]}$ This often leads to novel photo-products. $^{[4]}$

From the plethora of chemical reactions facilitated by electronic excitation, eliminative photochemical cyclisation has become one of the most important and widely used, because it provides an efficient route to the synthesis of polycyclic aromatic and heteroaromatic macromolecules. These molecules function as organic semiconductors, making them well suited for use in technological applications such as in organic light emitting diodes, ${ }^{[5-11]}$ organic solar cells, ${ }^{[12-16]}$ organic field effect transistors, ${ }^{[17-21]}$ liquid crystals ${ }^{[22-25]}$ and sensors. ${ }^{[26-28]}$

[a] A. D. D. Wonanke, Dr. J. L. Ferguson, Dr. C. M. Fitchett, Dr. D. L. Crittenden School of Physical and Chemical Sciences

University of Canterbury

Christchurch (New Zealand)

E-mail: deborah.crittenden@canterbury.ac.nz

(iD)

Supporting information and the ORCID identification number(s) for the au-

This manuscript is part of a special issue on chemistry in New Zealand. Click here to see the Table of Contents of the special issue.
Eliminative photocyclisation is an intramolecular light-activated aromatic ring forming reaction. Experimental evidence indicates that the reaction proceeds in two steps; planarisation followed by elimination. ${ }^{[29]}$ The photo-planarisation step involves a $6-\pi$ electrocyclisation of the reactant molecule into a cyclised intermediate, while the elimination step leads to ring aromatisation through the elimination of two atoms or functional groups adjacent to the newly formed bond. A simple illustration of this two-step mechanism is provided in Scheme 1.

Reactions in which $\mathrm{X}$ is a hydrogen atom require the presence of an oxidising agent to drive the elimination step. Such reactions are referred to as oxidative eliminative photocyclisations. On the other hand, reactions in which $\mathrm{X}$ is a suitable leaving group (alkoxy groups or halogens) are referred to as non-oxidative eliminative cyclisation since the elimination step can proceed spontaneously without an oxidising agent. ${ }^{[30]}$ In this paper, our focus will be on oxidative eliminative cyclisation reactions because they do not require substituted starting materials to be prepared and so are synthetically more convenient. Further, the use of oxidising agent to promote elimination allows an additional degree of synthetic control and further

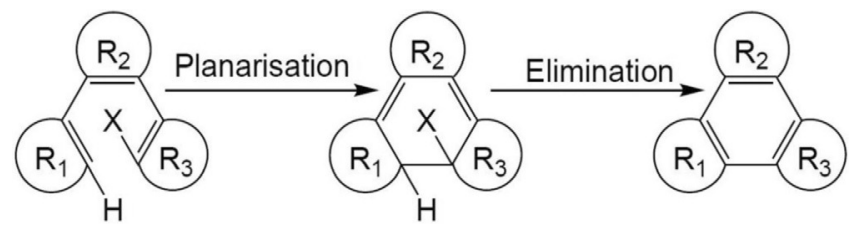

Scheme 1. General reaction Scheme for eliminative cyclisation, where $R_{1}, R_{2}$ $\mathrm{R}_{3}$ denote different ring systems. $\mathrm{X}$ represents a ring substituent which could be hydrogen or any suitable leaving group. 
potential for optimising reaction conditions to promote product formation. For brevity, photo-induced oxidative $6 \pi$ electrocyclisation reactions will henceforth be referred to simply as photocyclisations.

Although photocyclisation reactions are widely used synthetically, ${ }^{[31-35]}$ there is still a fundamental lack of understanding as to why certain molecules fail to yield products. Most experimental studies focus on optimising reaction conditionssolvent, oxidant, temperature-in an attempt to identify experimental conditions that will lead to product formation. However, recent computational studies have shown that the elimination step is thermodynamically favourable and can be kinetically enhanced by optimising reaction conditions, regardless of whether elimination proceeds in the excited state or following internal conversion back to the ground state. ${ }^{[30]}$ Further, if internal conversion is coupled to elimination, the whole process will proceed spontaneously and exothermically following photoexcitation and photoplanarisation. ${ }^{[30]}$

In either case, photoplanarisation is a necessary pre-condition for elimination, so it is important to be able to predict and/or control the process. Controlling the outcome of the photoplanarisation step is far harder than for elimination, because both the thermodynamic and kinetic favourability are determined primarily by changes in the molecule's electronic structure upon photo-excitation. In other words, photoplanarisability is an inherent property of a molecule that cannot be easily controlled by modifying reaction conditions.

In this joint experimental and computational study, our aim is to understand the factors that determine a molecule's ability to photoplanarise, and therefore photocyclise under appropriate reaction conditions. Experimentally, we will test a diverse set of polycyclic aromatic and heteroaromatic hydrocarbons for their ability to undergo photocyclisation under suitable oxidising conditions. Computationally, we will investigate how changes in molecular electronic structure upon photo-excitation affect a molecule's ability to photoplanarise and therefore photocyclise if reaction conditions are appropriately optimized to promote elimination. Changes in electronic structure will be characterised by analysing the forces that the changing electronic distribution induces at the atomic centres. Finally, we will assess computational predictions against experimental observations.

\section{Theory}

\subsection{Theoretical background}

Photoplanarisation is the first step in the photocyclisation process. Electronically, it can be viewed as a reorganisation of the $\pi$-electrons within the putative forming ring, with concomitant formation of a new $\sigma$ bond, creating a proto-aromatic system. Upon subsequent elimination of leaving groups adjacent to the newly formed ring, full aromaticity is realized. Geometrically, it can be viewed as a sterically-strained bond rotation, coupled to bond length changes indicative of aromatic ring formation.
For photoplanarisation to proceed, photo-induced changes in molecular electronic and geometric structure must be consistent with progress along this $6-\pi$ electrocyclisation reaction pathway. Previous studies have focussed on directly capturing changes in electron distribution upon photoexcitation, monitoring changes in quantities computed via Hückel molecular orbital theory such as free-valence indices, ${ }^{[36,37]}$ localisation energies, ${ }^{[36-38]}$ electronic overlap populations, ${ }^{[39,40]}$ and bond orders. $^{[41]}$

Although these reactivity predictors have been employed to good effect to predict regioselectivity and overall reactivity for polyaromatic hydrocarbons, applying them to heteroatom-containing systems becomes problematic. Hückel molecular orbital theory can be generalised to describe heteroaromatic systems, but extensive parameterisation is required. ${ }^{[42]}$ Separate parameters are required for every different atom and bond type, and atom type parameters can also vary according to the local bonding environment. ${ }^{[42]}$ Further, there is no single unique set of optimal parameters. ${ }^{[42]}$ As a consequence, no software exists to perform calculations of this type.

This suggests that a more sensible strategy would be to proceed directly to using modern electronic structure methods in combination with atomic population analysis schemes, to develop "modern-day" replacements. Unfortunately, atomic populations are not quantum observables, so all population analysis schemes are arbitrary. ${ }^{[43]}$ This makes it impossible to construct uniquely defined reactivity predictors. Further, any reactivity predictor will inherit the limitations and weaknesses of the underlying atomic population analysis Scheme and, in some cases, may not even be physically meaningful. ${ }^{[43]}$

Here, we contend that geometrically and energetically based indicators will provide a more robust, more generally applicable and less method-dependent way of assessing whether photo-induced changes in electronic structure are consistent with promoting planarisation, or not. Our approach relies on the fact that instantaneous changes in electron distributions upon photo-excitation at a given molecular conformation will induce forces on the atoms within the molecule, driving them to reorganise from their ground state conformation towards an excited state equilibrium structure. If these forces are consistent with the atoms moving to adopt a more planar and/or more aromatic ring-like system, then it is likely that photoplanarisation will proceed.

This suggests two possible metrics; the projection of the induced atomic forces along the torsional coordinate about which bond rotation must occur for the putative forming ring to planarise, or the projection of the induced atomic forces along bond vectors within the putative forming ring. The former is the most intuitive, as it directly answers the question-will the induced forces act to decrease the torsion angle and so planarise the molecule? However, this may be complicated by equal and/or opposing forces arising from steric strain. Analysing whether the bonds in the proposed forming ring become more aromatic is likely to be more computationally robust, because bond stretching vibrations tend to occur at high frequencies and low amplitudes, that is, are quite rigid motions due to high energy penalties for displacement from 
equilibrium. Therefore, instantaneous forces along bond vectors are likely to provide an accurate representation of how the structure will immediately adapt, uncomplicated by competing forces that may arise during longer time scale, largeamplitude motions.

Consider any two atoms, $i$ and $j$, within a putative forming ring whose positions are represented by the position vectors $r_{i}$

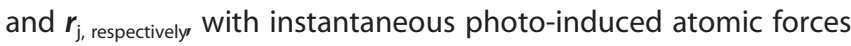
of $\boldsymbol{f}_{\mathrm{i}}$ and $\boldsymbol{f}_{\mathrm{j}}$ computed at the molecule's ground state equilibrium geometry. The bond vector between these two atoms is $\boldsymbol{r}_{\mathrm{i}, \mathrm{j}}=\boldsymbol{r}_{\mathrm{i}}-\boldsymbol{r}_{\mathrm{j}}$ and the corresponding force vector is $\boldsymbol{f}_{\mathrm{i}, \mathrm{j}}=\boldsymbol{f}_{\mathrm{i}}-\boldsymbol{f}_{\mathrm{j} \text {. }}$.

Then the component of the force that acts directly along the bond is simply:

$F_{i, j}^{\text {proj }}=\frac{\mathbf{f}_{i, j} \cdot \mathbf{r}_{i, j}}{\left\|\mathbf{r}_{i, j}\right\|}$

Finally, it remains to consider how the photo-induced forces affect all bonds within the photocyclising ring system, excluding the forming bond. To be consistent with aromatization, atom-pairs that are $\sigma$-bonded in the ground state would acquire negative projected forces in the excited state, indicating a driving force towards these bonds becoming shorter. Conversely, $\pi$-bonded atom-pairs would acquire positive projected forces, indicative of bond lengthening.

If these conditions are fulfilled, then the total projected excited state "bond aromatization force" is computed as:

$F_{\pi, \sigma}^{*}=\sum_{i=1}^{n-1} \sum_{j=i+1}^{n}\left\|F_{i, j}^{\text {proj }}\right\|$

where the sums run over all unique atom-pairs in the putative forming ring that are bonded in the ground state.

\subsection{Computational details}

Ground state geometries are optimised for isolated, gas phase species at B3LYP/6-31G* ${ }^{[44-48]}$ The B3LYP functional is chosen because it is well-parameterised for organic systems, and is one of the few functionals for which analytic gradients and Hessians are available in both ground and excited states. The 6-31G* basis set is used because it is the basis in which the B3LYP functional was parameterized and so affords near optimal error cancellation between BSSE and dispersion contributions to the energy that are not explicitly accounted for. ${ }^{[49]}$ All excited state gradients are computed for the first singlet excited state at TD-B3LYP/6-31G*, unless otherwise specified. All calculations are performed using the Q-Chem5.0 software package. Our force projection code can be freely downloaded from http://github.com/dlc62/force-projection.

\section{Experimental Results and Discussion}

To properly benchmark computational predictions against experiment, a comprehensive data set of photocyclisation reaction conditions and outcomes is required. Photocyclisation reaction conditions and outcomes have been compiled from the literature in Table 1 for a series of compounds based upon (1) stilbenyl, ${ }^{[29,31,50]}$ (2) phenanthryl, ${ }^{[50,51]}$ (3) acrylamide, ${ }^{[52]}$ (4) furyl, ${ }^{[50]}$ (5) thiophenyl, ${ }^{[50]}$ (6) pyrrolyl ${ }^{[53,54]}$ and (9) benzyl ${ }^{[50,53]}$ scaffolds illustrated in Figure 1.<smiles>[R]C([R])=C([R])[R]</smiles>

1. Stilbenyl<smiles>[R]c1cc2c([R])c([R])c3ccc[Y]c3c2c([R])c1[R]</smiles>

2. Phenanthryl<smiles>[R]c1oc([R])c([R])c1[R]</smiles>

4. Furyl<smiles>[R]c1sc([R])c([R])c1[R]</smiles>

5. Thiophenyl<smiles></smiles>

3. Acrylamide<smiles>[R]c1c([R])n([X])c2ccccc12</smiles>

7. Indolyl<smiles>[Y]c1ccc2c(c1)c1cc([Y])ccc1c1c2c([R2])c([R7])n1[X]</smiles>

8. Dibenzo[e,g]indolyl<smiles>[R]c1c([R])c([R])n([X])c1[R]</smiles>

6. Pyrrolyl
Figure 1. Scaffold of molecules present in our dataset where A, B, and C represent ring constituents, $\mathrm{R}$ represent ring substituents that are potentially involved in photocyclisation, $X$ and $Y$ represent electronically active substituents, and the identity of these groups are defined in 1. For each molecule, photocyclisation can occur between ring substituents, between a ring substituent and the scaffold or between the scaffold atoms (...).

To extend the breadth and scope of our reference data set, we have also experimentally investigated photocyclisation of a novel set of 2,3-diaryl substituted indoles, 7. First, a series of substituted $\mathrm{N}$-ethyl or $\mathrm{N}$-benzyl indoles were synthesised by alkylation of the appropriate $\mathrm{N}-\mathrm{H}$ indole to give $\mathbf{7 a - 7} \mathbf{h}$ (see ESI for details). Scaffolds of these molecules are illustrated in Figure 1 and their substituents listed in Table 1.

Photocyclisation reactions for $\mathbf{7 a - 7 h}$ were carried out in a Rayonet photoreactor irradiating with $300 \mathrm{~nm}$ light over 12 hours. All reactions were performed in a quartz tube with toluene as the solvent, 1.1 molar equivalents of $\mathrm{I}_{2}$ and $5 \mathrm{~mL}$ of propylene oxide. ${ }^{[33]}$ A constant stream of argon was bubbled through the reaction solution for the duration of the reaction to remove oxygen. The dibenzo[ $a, c]$ carbazole products, $10 \mathrm{a}-$ $10 \mathrm{~h}$ (Figure 2), were isolated in moderate to good yields after column chromatography (see Table 1), and have been fully characterised using NMR spectroscopy and electrospray mass spectrometry (ES-MS).

Cyclisation was confirmed by examination of ${ }^{1} \mathrm{H}$ NMR spectra, which showed considerable desymmetrisation due to aryl ring rotation (see ESI for spectra). This was also associated with a general shift of the resonances to more downfield positions, 
Table 1. Table of photocyclisation yields and experimental conditions of data compiled from this studies and published literatures. The molecular scaffolds and substituent patterns for these molecules are illustrated in 1 and the identities of functional groups are defined below.

\begin{tabular}{|c|c|c|c|c|c|c|}
\hline & Molecules & Solvent & Oxidant & Forming ring & Yields [\%] & Ref \\
\hline \multicolumn{7}{|c|}{ 1.Stilbenyl } \\
\hline a & $\mathrm{R}_{1}=\mathrm{R}_{2}=2$-furyl, $\mathrm{R}_{3}=\mathrm{R}_{4}=\mathrm{H}$ & ethanol & $\mathrm{CuCl}_{2} / \mathrm{I}_{2}$ & $R_{1}-R_{2}$ & 24 & [50] \\
\hline b & $\mathrm{R}_{1}=\mathrm{R}_{2}=2$-thiophenyl, $\mathrm{R}_{3}=\mathrm{R}_{4}=\mathrm{H}$ & cyclohexane & $\mathrm{I}_{2} / \mathrm{O}_{2}$ & $R_{1}-R_{2}$ & 90 & [31] \\
\hline c & $\mathrm{R}_{1}=\mathrm{R}_{2}=$ phenyl, $\mathrm{R}_{3}=\mathrm{R}_{4}=\mathrm{H}$ & cyclohexane & $\mathrm{I}_{2} / \mathrm{O}_{2}$ & $R_{1}-R_{2}$ & 82 & [29] \\
\hline d & $R_{1}=R_{2}=R_{3}=R_{4}=$ phenyl & cyclohexane & $\mathrm{I}_{2} / \mathrm{O}_{2}$ & $R_{1}-R_{2}$ & 88 & [50] \\
\hline e & $\mathrm{R}_{1}=\mathrm{R}_{2}=\mathrm{R}_{3}=\mathrm{R}_{4}=$ 2-thiophenyl & toluene & $\mathrm{I}_{2}$ & $R_{1}-R_{2}$ & 14 & [51] \\
\hline $\mathrm{f}$ & $\mathrm{R}_{1}=\mathrm{R}_{2}=$ 2-thiophenyl, $\mathrm{R}_{3}=\mathrm{R}_{4}=$ phenyl & toluene & $\mathrm{I}_{2}$ & $\mathrm{R}_{1}-\mathrm{R}_{2}$ & 68 & [51] \\
\hline \multicolumn{7}{|c|}{ 2. Phenanthryl, $\mathrm{A}=\mathrm{CH}$} \\
\hline a & $\mathrm{R}_{1}=\mathrm{R}_{2}=$ phenyl, $\mathrm{R}_{3}=\mathrm{R}_{4}=\mathrm{R}_{5}=\mathrm{H}$ & cyclohexane & $\mathrm{I}_{2}$ & - & - & [50] \\
\hline b & $R_{1}=R_{2}=$ 2-thiophenyl, $R_{3}=R_{4}=R_{5}=H$ & toluene & $\mathrm{I}_{2} / \mathrm{O}_{2}$ & $R_{1}-R_{2}$ & 69 & [51] \\
\hline c & $\mathrm{R}_{1}=\mathrm{R}_{2}=\mathrm{R}_{5}=\mathrm{H}, \mathrm{R}_{3}=\mathrm{R}_{4}=$ phenyl & benzene & $\mathrm{I}_{2}$ & - & - & [50] \\
\hline d & $\mathrm{R}_{1}=\mathrm{R}_{2}=\mathrm{R}_{3}=\mathrm{H}, \mathrm{R}_{4}=\mathrm{R}_{5}=$ phenyl & benzene & $\mathrm{I}_{2}$ & - & - & [50] \\
\hline e & $\mathrm{R}_{1}=\mathrm{R}_{2}=\mathrm{R}_{3}=\mathrm{R}_{4}=\mathrm{H}, \mathrm{R}_{5}=$ phenyl & benzene & $\mathrm{I}_{2}$ & $\mathrm{~A}-\mathrm{R}_{5}$ & 46 & [50] \\
\hline$f$ & $\mathrm{R}_{1}=\mathrm{R}_{2}=\mathrm{R}_{3}=\mathrm{R}_{4}=\mathrm{H}, \mathrm{R}_{5}=$ 2-naphthyl & benzene & $\mathrm{I}_{2}$ & $\mathrm{~A}-\mathrm{R}_{5}$ & 35 & [50] \\
\hline \multicolumn{7}{|c|}{ 3. Acrylamides } \\
\hline a & $\mathrm{A}=\mathrm{B}=\mathrm{CH}, \mathrm{C}=\mathrm{N}, \mathrm{X}=\mathrm{H}$ & benzene/acetic acid & $\mathrm{SeO}_{2}$ & $\ldots$ & 17 & [52] \\
\hline b & $\mathrm{A}=\mathrm{B}=\mathrm{CH}, \mathrm{C}=\mathrm{N}, \mathrm{X}=\mathrm{Me}$ & benzene/acetic acid & $\mathrm{SeO}_{2}$ & $\ldots$ & 78 & [52] \\
\hline c & $\mathrm{A}=\mathrm{C}=\mathrm{CH}, \mathrm{B}=\mathrm{N}, \mathrm{X}=\mathrm{Me}$ & benzene/acetic acid & $\mathrm{SeO}_{2}$ & $\ldots$ & 22 & [52] \\
\hline d & $\mathrm{A}=\mathrm{CH}, \mathrm{B}=\mathrm{N}, \mathrm{C}=\mathrm{CCl}, \mathrm{X}=\mathrm{Me}$ & benzene/acetic acid & $\mathrm{SeO}_{2}$ & $\ldots$ & 25 & [52] \\
\hline e & $\mathrm{A}=\mathrm{N}, \mathrm{B}=\mathrm{C}=\mathrm{CH}, \mathrm{X}=\mathrm{Me}$ & benzene/acetic acid & $\mathrm{SeO}_{2}$ & $\ldots$ & 72 & [52] \\
\hline \multicolumn{7}{|c|}{ 4. Furyl } \\
\hline a & $\mathrm{R}_{1}=\mathrm{R}_{2}=$ phenyl, $\mathrm{R}_{3}=\mathrm{R}_{4}=\mathrm{H}$ & benzene & $\mathrm{O}_{2}$ & $R_{1}-R_{2}$ & 40 & [50] \\
\hline b & $\mathrm{R}_{1}=\mathrm{R}_{2}=\mathrm{R}_{3}=\mathrm{R}_{4}=$ phenyl & benzene & $\mathrm{O}_{2}$ & - & $\mathrm{p}$ & [50] \\
\hline \multicolumn{7}{|c|}{ 5. Thiophenyl } \\
\hline a & $\mathrm{R}_{1}=\mathrm{R}_{2}=$ phenyl, $\mathrm{R}_{3}=\mathrm{R}_{4}=\mathrm{H}$ & benzene & $\mathrm{O}_{2}$ & $\mathrm{R}_{1}-\mathrm{R}_{2}$ & 70 & [50] \\
\hline b & $\mathrm{R}_{1}=\mathrm{R}_{2}=\mathrm{R}_{3}=\mathrm{R}_{4}=$ phenyl & benzene & $\mathrm{O}_{2}$ & - & $\mathrm{p}$ & [50] \\
\hline \multicolumn{7}{|c|}{ 6. Pyrrolyl } \\
\hline a & $\mathrm{R}_{1}=\mathrm{R}_{2}=\mathrm{R}_{3}=\mathrm{R}_{4}=$ phenyl, $\mathrm{X}=\mathrm{H}$ & benzene & $\mathrm{O}_{2}$ & - & - & [53] \\
\hline b & $\mathrm{R}_{1}=\mathrm{R}_{2}=\mathrm{R}_{3}=\mathrm{R}_{4}=$ phenyl, $\mathrm{X}=\mathrm{Et}$ & toluene & $\mathrm{I}_{2} / \mathrm{PrO}$ & $R_{1}-R_{2}$ & 73 & [54] \\
\hline \multicolumn{7}{|c|}{ 7. Indolyl } \\
\hline a & $\mathrm{R}_{1}=\mathrm{R}_{2}=$ phenyl, $\mathrm{X}=\mathrm{Et}$ & toluene & $\mathrm{I}_{2} / \mathrm{PrO}$ & $R_{1}-R_{2}$ & 64 & This work \\
\hline b & $\mathrm{R}_{1}=\mathrm{R}_{2}=$ 4-methoxyphenyl, $\mathrm{X}=\mathrm{Et}$ & toluene & $\mathrm{I}_{2} / \mathrm{PrO}$ & $\mathrm{R}_{1}-\mathrm{R}_{2}$ & 73 & This work \\
\hline c & $\mathrm{R}_{1}=\mathrm{R}_{2}=4$-methylphenyl, $\mathrm{X}=\mathrm{Et}$ & toluene & $\mathrm{I}_{2} / \mathrm{PrO}$ & $R_{1}-R_{2}$ & 60 & This work \\
\hline d & $\mathrm{R}_{1}=\mathrm{R}_{2}=$ 4-tert-butylphenyl, $\mathrm{X}=\mathrm{Et}$ & toluene & $\mathrm{I}_{2} / \mathrm{PrO}$ & $R_{1}-R_{2}$ & 89 & This work \\
\hline e & $\mathrm{R}_{1}=\mathrm{R}_{2}=$ phenyl, $\mathrm{X}=\mathrm{Bz}$ & toluene & $\mathrm{I}_{2} / \mathrm{PrO}$ & $R_{1}-R_{2}$ & 84 & This work \\
\hline$f$ & $\mathrm{R}_{1}=\mathrm{R}_{2}=$ 4-methoxyphenyl, $\mathrm{X}=\mathrm{Bz}$ & toluene & $\mathrm{I}_{2} / \mathrm{PrO}$ & $R_{1}-R_{2}$ & 62 & This work \\
\hline g & $\mathrm{R}_{1}=\mathrm{R}_{2}=$ 4-methylphenyl, $\mathrm{X}=\mathrm{Bz}$ & toluene & $\mathrm{I}_{2} / \mathrm{PrO}$ & $R_{1}-R_{2}$ & 56 & This work \\
\hline $\mathrm{h}$ & $R_{1}=R_{2}=4$-tert-butylphenyl, $X=B z$ & toluene & $\mathrm{I}_{2} / \mathrm{PrO}$ & $R_{1}-R_{2}$ & 78 & This work \\
\hline \multicolumn{7}{|c|}{ 8. Dibenzo[e,g]indole } \\
\hline a & $\mathrm{R}_{1}=\mathrm{R}_{2}=$ phenyl, $\mathrm{X}=\mathrm{Et}, \mathrm{Y}=\mathrm{H}$ & toluene & $\mathrm{I}_{2} / \mathrm{PrO}$ & - & - & This work \\
\hline b & $R_{1}=R_{2}=4$-methylphenyl, $X=E t, Y=M e$ & toluene & $\mathrm{I}_{2} / \mathrm{PrO}$ & - & - & This work \\
\hline c & $\mathrm{R}_{1}=\mathrm{R}_{2}=4$-tert-butylphenyl, $\mathrm{X}=\mathrm{Et}, \mathrm{Y}=t$-Bu & toluene & $\mathrm{I}_{2} / \mathrm{PrO}$ & - & - & This work \\
\hline \multicolumn{7}{|c|}{ 9. Benzyl } \\
\hline a & $\mathrm{R}_{1}=\mathrm{R}_{2}=$ phenyl, $\mathrm{R}_{3}=\mathrm{R}_{4}=\mathrm{R}_{5}=\mathrm{R}_{6}=\mathrm{H}$ & benzene & $\mathrm{I}_{2} / \mathrm{N}_{2}$ & $R_{1}-R_{2}$ & 88 & [50] \\
\hline b & $\mathrm{R}_{1}=\mathrm{R}_{2}=\mathrm{R}_{3}=$ phenyl, $\mathrm{R}_{4}=\mathrm{R}_{5}=\mathrm{R}_{6}=\mathrm{H}$ & benzene & $\mathrm{I}_{2} / \mathrm{N}_{2}$ & $R_{1}-R_{2}-R_{3}$ & 21 & [53] \\
\hline c & $\mathrm{R}_{1}=\mathrm{R}_{4}=\mathrm{H}, \mathrm{R}_{2}=\mathrm{R}_{3}=\mathrm{R}_{5}=\mathrm{R}_{6}=$ phenyl & benzene & $\mathrm{I}_{2}$ & $\mathrm{R}_{2}-\mathrm{R}_{3}$ & $\mathrm{p}$ & [53] \\
\hline d & $\mathrm{R}_{1}=\mathrm{R}_{2}=\mathrm{R}_{3}=\mathrm{R}_{4}=$ phenyl, $\mathrm{R}_{5}=\mathrm{R}_{6}=\mathrm{H}$ & benzene & $\mathrm{I}_{2}$ & - & - & [53] \\
\hline e & $\mathrm{R}_{1}=\mathrm{R}_{2}=\mathrm{R}_{3}=\mathrm{R}_{4}=\mathrm{R}_{5}=$ phenyl, $\mathrm{R}_{6}=\mathrm{H}$ & benzene & $\mathrm{I}_{2}$ & - & - & [53] \\
\hline$f$ & $R_{1}=R_{2}=R_{3}=R_{4}=R_{5}=R_{6}=$ phenyl & benzene & $\mathrm{I}_{2}$ & - & - & [53] \\
\hline
\end{tabular}

with the most downfield protons in 10 ranging from 8.21$8.94 \mathrm{ppm}$ (compared to 7.76-7.85 ppm for 7). The formation of two singlets for the protons adjacent to the new bond for $\mathbf{1 0} \mathbf{b}-\mathbf{d}$ and $\mathbf{1 0} \mathbf{f}-\mathbf{h}$ is indicative of the loss of these protons during the cyclodehydrogenation reaction. The protons of the $\mathrm{N}$-alkylating group were also affected, with the protons of the attaching methylene shifted to $\approx 4.9 \mathrm{ppm}$ (for $10 \mathrm{a}-10 \mathrm{~d}$ ) and $\approx 6.0 \mathrm{ppm}$ (for $10 \mathrm{e}-10 \mathrm{~h}$ ) from $\approx 4.1 \mathrm{ppm}$ and $\approx 5.3 \mathrm{ppm}$, respectively. These changes are indicative of the increased aro- matic area of 10, and the associated increase in the ring anisotropy. ${ }^{[54]}$

We were able to grow single crystals of $10 \mathrm{c}, 10 \mathrm{f}$ and $10 \mathrm{~g}$, and these were characterised using single crystal X-ray diffraction, along with the structures of $\mathbf{7} \mathbf{a}, \mathbf{7} \mathbf{d}$, and $\mathbf{7} \mathbf{h}$ (see the Supporting Information). The molecules all crystallised with one molecule in the asymmetric unit, as exemplified by the structure of $10 \mathrm{f}$ shown in Figure 3 (see ESI for structures of $10 \mathrm{c}$ and $10 \mathrm{~g}$ ). 


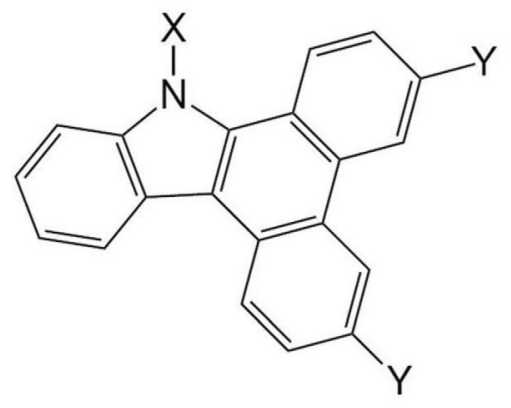

(a) $X=E t, Y=H$

(b) $X=E t, Y=O M e$

(c) $X=E t, Y=M e$

(d) $X=E t, Y=t B u$

(e) $\mathrm{X}=\mathrm{Bz}, \mathrm{Y}=\mathrm{H}$

(f) $X=B z, Y=O M e$

(g) $\mathrm{X}=\mathrm{Bz}, \mathrm{Y}=\mathrm{Me}$

(h) $X=B z, Y=t B u$

Figure 2. Dibenzo[a,c]carbazoles (10) formed by oxidative photocyclisation of 2,3-diaryl substituted indoles (7).

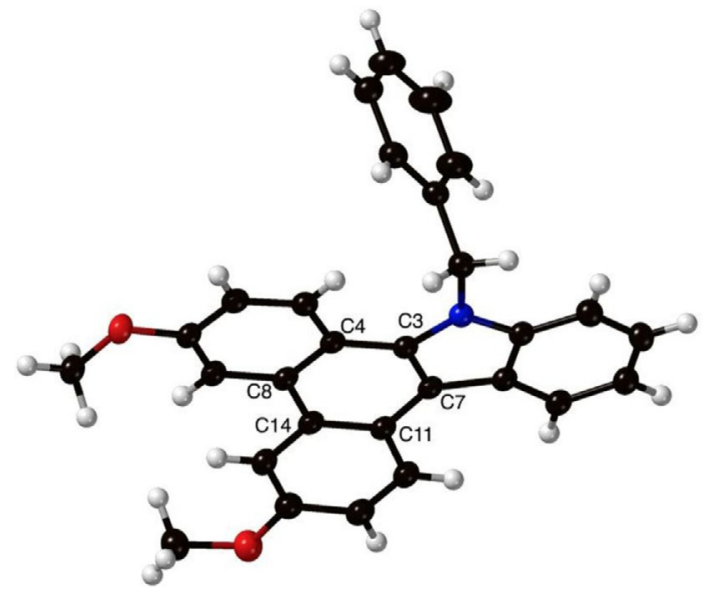

Figure 3. Crystallographic molecular structure of $10 \mathrm{f}$, showing the newly formed ring system. Thermal ellipsoids are shown at the $50 \%$ level. Selected bond lengths ( $\AA$ ): C11-C14 1.417(2), C3-C4 1.441(2), C3-C7 1.392(2), C4-C8 $1.420(2), C 7-C 11$ 1.438(2), C8-C14 1.467(2).

The structures show the cyclised products, with newly formed $\mathrm{C}-\mathrm{C}$ bond lengths of 1.465(2) $\AA$ (10 c), 1.467(2) $\AA$ (10 f) and $1.462(2) \AA(\mathbf{1 0} \mathbf{g})$. The enlarged aromatic dibenzo[a,c]carbazole ring systems are remarkably planar, with RMS deviations of $0.045 \AA$ (10 c), $0.066 \AA$ (10 f) and $0.092 \AA(10 \mathrm{~g})$. This planarity forces the $N$-ethyl and $N$-benzyl groups to be almost perpendicular to the plane, with torsion angles of $97.7^{\circ}$ (2) (10 c), $104.5^{\circ}$ (2) (10 f) and $90.8(2)^{\circ}(\mathbf{1 0} \mathbf{g})$. Due to the large area of the dibenzo[ $a, c]$ carbazole ring, the structures all exhibit faceto-face interactions in the solid state, with the closest approach being 3.475(1) $\AA$ (10 c), 3.379(1) $\AA$ (10 f) and 3.551(1) $\AA$ $(\mathbf{1 0} \mathrm{g})$.

We have also investigated the propensity of the structurallyrelated dibenzo[e,g]indoles, $\mathbf{8}$, to undergo cyclisation under similar reaction conditions. However, no evidence of cyclised product was observed via either NMR or ES-MS. However, we have previously shown that dibenzo[e,g]indoles can themselves be formed by photocyclisation from appropriately substituted pyrroles, $6 .^{[54]}$

In total, we report reaction conditions and outcomes for 40 different potentially photocyclisable systems in Table 1 , repre- senting a diverse range of molecular scaffolds and substitution patterns.

\section{Computational Results and Discussion}

Total photo-induced bond aromatisation forces around all possible rings that may form within reactants are reported in Table 2. The complete set of molecular structures and first singlet excited state transition energies are available in the Electronic Supporting Information. Most transition energies are predicted to fall in the near-UV range $(300-400 \mathrm{~nm}, 3.1-$ $4.1 \mathrm{eV})$, although some fall in the experimentally accessible 250-300 nm mid-UV range (4.1-4.9 eV), primarily molecules in the benzyl and acrylamide classes.

\subsection{Correlating predicted photoplanarisation with observed photocyclisation}

There are 6 classes of molecules within our data set for which photocyclised products are consistently formed, regardless of substitution pattern: stilbenyl, acrylamide, furyl, thiophenyl, pyrrolyl and indolyl. In all cases, the total bond aromatisation force is larger than $0.15 \mathrm{E}_{\mathrm{h}} / \mathrm{a}_{0}$ around the photocyclising ring system that leads to the observed isomeric product. In the cases where separation and characterisation of products was not attempted, at least one had a corresponding value of $F_{\pi, \sigma}^{*}$ above 0.15 . Figure 4 illustrates photo-induced atomic forces for a prototypical photocyclisable system, cis-stilbene (1 c). They clearly act to elongate $\pi$ bonds and shorten $\sigma$ bonds, that is, make the photocyclising ring system more aromatic.
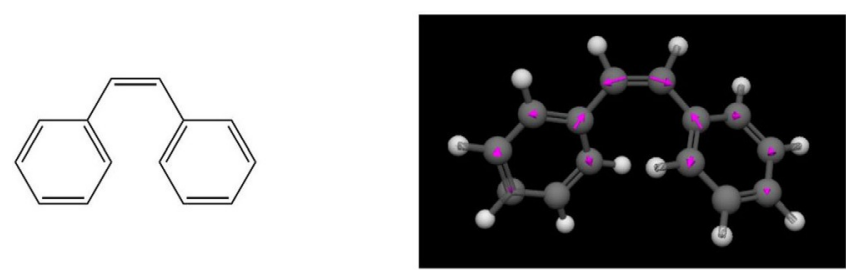

Figure 4. Molecular structure of cis-stilbene (left) and photo-induced atomic forces (right).

Despite trialling a range of experimental conditions for a series of molecules with different substitution patterns, no photocyclised product could be obtained from dibenzo[e,g]indoles. Computationally, these molecules are found to have low bond aromatisation forces $\left(0.04 \mathrm{E}_{\mathrm{h}} / \mathrm{a}_{0}\right)$. Photo-induced atomic forces for a prototypical dibenzo[e,g]indole system $(\mathbf{8} \mathbf{a})$ are illustrated in Figure 5. This Figure shows that the electronic transition occurs solely on the dibenzo[e,g]indole scaffold, and so does not promote aromatization or drive photoplanarisation. If the photoplanarised intermediate cannot be formed, then the elimination step is unlikely to proceed. This is consistent with experimental observations that no product can be formed.

For substituted benzyl and phenanthryl systems, experimental outcomes are dependent on substitution pattern. Heavily 
Table 2. Total photo-induced bond aromatisation forces, $F^{*}{ }_{\pi, 0}$, along al possible photocyclisation pathways for all molecules in our data set. For clarity, experimentally-determined yields are reproduced from Table 1.

\begin{tabular}{|c|c|c|c|}
\hline Molecules & $\begin{array}{l}\text { Forming } \\
\text { ring }\end{array}$ & $\begin{array}{l}\mathrm{F}_{\pi, \sigma}^{*}\left(\mathrm{E}_{\mathrm{h}} /\right. \\
\left.\mathrm{a}_{0}\right)\end{array}$ & $\begin{array}{l}\text { Yield } \\
{[\%]}\end{array}$ \\
\hline \multicolumn{4}{|l|}{ 1. Stilbenyl } \\
\hline a $\mathrm{R}_{1}=\mathrm{R}_{2}=2$-furyl, $\mathrm{R}_{3}=\mathrm{R}_{4}=\mathrm{H}$ & $\mathrm{R}_{1}-\mathrm{R}_{2}$ & 0.49 & 24 \\
\hline b $R_{1}=R_{2}=$ 2-thiophenyl, $R_{3}=R_{4}=H$ & $\mathrm{R}_{1}-\mathrm{R}_{2}$ & 0.49 & 90 \\
\hline c $\mathrm{R}_{1}=\mathrm{R}_{2}=$ phenyl, $\mathrm{R}_{3}=\mathrm{R}_{4}=\mathrm{H}$ & $\mathrm{R}_{1}-\mathrm{R}_{2}$ & 0.47 & 82 \\
\hline d $R_{1}=R_{2}=R_{3}=R_{4}=$ phenyl & $\mathrm{R}_{1}-\mathrm{R}_{2}=\mathrm{R}_{3}-\mathrm{R}_{4}$ & 0.32 & 88 \\
\hline e $\mathrm{R}_{1}=\mathrm{R}_{2}=\mathrm{R}_{3}=\mathrm{R}_{4}=$ 2-thiophenyl & $R_{1}-R_{2}=R_{3}-R_{4}$ & 0.30 & 14 \\
\hline f $R_{1}=R_{2}=$ 2-thiophenyl, & $\mathrm{R}_{1}-\mathrm{R}_{2}$ & 0.34 & 68 \\
\hline $\begin{array}{l}\mathrm{R}_{3}=\mathrm{R}_{4}=\text { phenyl } \\
\text { 2. Phenanthryl, } \mathrm{A}=\mathrm{CH}\end{array}$ & $\mathrm{R}_{3}-\mathrm{R}_{4}$ & 0.25 & - \\
\hline a $R_{1}=R_{2}=$ phenyl, $R_{3}=R_{4}=R_{5}=H$ & $\mathrm{R}_{1}-\mathrm{R}_{2}$ & 0.12 & - \\
\hline $\begin{aligned} \text { b } R_{1} & =R_{2}=2 \text {-thiophenyl, } \\
R_{3} & =R_{4}=R_{5}=H\end{aligned}$ & $\mathrm{R}_{1}-\mathrm{R}_{2}$ & 0.30 & 69 \\
\hline c $\mathrm{R}_{1}=\mathrm{R}_{2}=\mathrm{R}_{5}=\mathrm{H}, \mathrm{R}_{3}=\mathrm{R}_{4}=$ phenyl & $\mathrm{R}_{3}-\mathrm{R}_{5}$ & 0.09 & - \\
\hline d $R_{1}=R_{2}=R_{3}=H, R_{4}=R_{5}=$ phenyl & $\mathrm{R}_{3}-\mathrm{R}_{4}$ & 0.09 & - \\
\hline e $\mathrm{R}_{1}=\mathrm{R}_{2}=\mathrm{R}_{3}=\mathrm{R}_{4}=\mathrm{H}, \mathrm{R}_{5}=$ phenyl & $\mathrm{A}-\mathrm{R}_{5}$ & 0.11 & 46 \\
\hline $\begin{array}{l}\text { f } R_{1}=R_{2}=R_{3}=R_{4}=H, R_{5}=2 \text {-naphthyl } \\
\text { 3. Acrylamide }\end{array}$ & $\mathrm{A}-\mathrm{R}_{5}$ & 0.11 & 35 \\
\hline a $\mathrm{A}=\mathrm{B}=\mathrm{CH}, \mathrm{C}=\mathrm{N}, \mathrm{X}=\mathrm{H}$ & $\cdots$ & 0.29 & 17 \\
\hline b $\mathrm{A}=\mathrm{B}=\mathrm{CH}, \mathrm{C}=\mathrm{N}, \mathrm{X}=\mathrm{Me}$ & $\ldots$ & 0.29 & 78 \\
\hline c $\mathrm{A}=\mathrm{C}=\mathrm{CH}, \mathrm{B}=\mathrm{N}, \mathrm{X}=\mathrm{Me}$ & $\ldots$ & 0.28 & 53 \\
\hline d $\mathrm{A}=\mathrm{CH}, \mathrm{B}=\mathrm{N}, \mathrm{C}=\mathrm{CCl}, \mathrm{X}=\mathrm{Me}$ & $\ldots$ & 0.27 & 25 \\
\hline $\begin{array}{l}\text { e } \mathrm{A}=\mathrm{N}, \mathrm{B}=\mathrm{C}=\mathrm{CH}, \mathrm{X}=\mathrm{Me} \\
\text { 4. Furyl }\end{array}$ & $\cdots$ & 0.26 & 72 \\
\hline a $\mathrm{R}_{1}=\mathrm{R}_{2}=$ phenyl, $\mathrm{R}_{3}=\mathrm{R}_{4}=\mathrm{H}$ & $\begin{array}{l}\mathrm{R}_{1}-\mathrm{R}_{2} \\
\mathrm{R}_{1}-\mathrm{R}_{2}\end{array}$ & $\begin{array}{l}0.40 \\
0.21\end{array}$ & 40 \\
\hline b $\mathrm{R}_{1}=\mathrm{R}_{2}=\mathrm{R}_{3}=\mathrm{R}_{4}=$ phenyl & $\begin{array}{l}R_{2}-R_{3} \\
R_{3}-R_{4}\end{array}$ & $\begin{array}{l}0.11 \\
0.21\end{array}$ & $\mathrm{p}$ \\
\hline \multicolumn{4}{|l|}{ 5. Thiophenyl } \\
\hline a $\mathrm{R}_{1}=\mathrm{R}_{2}=$ phenyl, $\mathrm{R}_{3}=\mathrm{R}_{4}=\mathrm{H}$ & $\begin{array}{l}R_{1}-R_{2} \\
R_{1}-R_{2}\end{array}$ & $\begin{array}{l}0.39 \\
0.21\end{array}$ & 70 \\
\hline b $\mathrm{R}_{1}=\mathrm{R}_{2}=\mathrm{R}_{3}=\mathrm{R}_{4}=$ phenyl & $\begin{array}{l}\mathrm{R}_{2}-\mathrm{R}_{3} \\
\mathrm{R}_{3}-\mathrm{R}_{4}\end{array}$ & $\begin{array}{l}0.11 \\
0.21\end{array}$ & $\mathrm{p}$ \\
\hline \multicolumn{4}{|l|}{ 6. Pyrrolyl } \\
\hline & $\mathrm{R}_{1}-\mathrm{R}_{2}$ & 0.17 & \\
\hline \multirow[t]{3}{*}{ a $\mathrm{R}_{1}=\mathrm{R}_{2}=\mathrm{R}_{3}=\mathrm{R}_{4}=$ phenyl, $\mathrm{X}=\mathrm{H}$} & $\mathrm{R}_{2}-\mathrm{R}_{3}$ & 0.09 & $\mathrm{p}$ \\
\hline & $\mathrm{R}_{3}-\mathrm{R}_{4}$ & 0.17 & \\
\hline & $\mathrm{R}_{1}-\mathrm{R}_{2}$ & 0.21 & 73 \\
\hline \multirow[t]{2}{*}{ b $\mathrm{R}_{1}=\mathrm{R}_{2}=\mathrm{R}_{3}=\mathrm{R}_{4}=$ phenyl, $\mathrm{X}=\mathrm{Et}$} & $\mathrm{R}_{2}-\mathrm{R}_{3}$ & 0.09 & \\
\hline & $\mathrm{R}_{3}-\mathrm{R}_{4}$ & 0.15 & \\
\hline a $\mathrm{R}_{1}=\mathrm{R}_{2}=$ phenyl, $\mathrm{X}=\mathrm{Et}$ & $\mathrm{R}_{1}-\mathrm{R}_{2}$ & 0.30 & 64 \\
\hline b $\mathrm{R}_{1}=\mathrm{R}_{2}=$ 4-methoxyphenyl, $\mathrm{X}=\mathrm{Et}$ & $\mathrm{R}_{1}-\mathrm{R}_{2}$ & 0.35 & 60 \\
\hline c $\mathrm{R}_{1}=\mathrm{R}_{2}=4$-methylphenyl, $\mathrm{X}=\mathrm{Et}$ & $\mathrm{R}_{1}-\mathrm{R}_{2}$ & 0.31 & 87 \\
\hline d $\mathrm{R}_{1}=\mathrm{R}_{2}=4$-tert-butylphenyl, $\mathrm{X}=\mathrm{Et}$ & $\mathrm{R}_{1}-\mathrm{R}_{2}$ & 0.33 & 89 \\
\hline e $\mathrm{R}_{1}=\mathrm{R}_{2}=$ phenyl, $\mathrm{X}=\mathrm{Bz}$ & $\mathrm{R}_{1}-\mathrm{R}_{2}$ & 0.30 & 84 \\
\hline f $\mathrm{R}_{1}=\mathrm{R}_{2}=4$-methoxyphenyl, $\mathrm{X}=\mathrm{Bz}$ & $\mathrm{R}_{1}-\mathrm{R}_{2}$ & 0.33 & 62 \\
\hline g $R_{1}=R_{2}=4$-methylphenyl, $X=B z$ & $\mathrm{R}_{1}-\mathrm{R}_{2}$ & 0.32 & 56 \\
\hline $\begin{array}{l}\text { h } R_{1}=R_{2}=4 \text {-tert-butylphenyl, } X=B z \\
\text { 8. Dibenzo[e,g]indole }\end{array}$ & $\mathrm{R}_{1}-\mathrm{R}_{2}$ & 0.33 & 78 \\
\hline a $\mathrm{R}_{1}=\mathrm{R}_{2}=$ phenyl, $\mathrm{X}=\mathrm{Et}, \mathrm{Y}=\mathrm{H}$ & $\mathrm{R}_{1}-\mathrm{R}_{2}$ & 0.04 & - \\
\hline b $\begin{array}{l}R_{1}=R_{2}=4 \text {-methylphenyl, } X=E t \\
Y=H\end{array}$ & $\mathrm{R}_{1}-\mathrm{R}_{2}$ & 0.04 & - \\
\hline c $\begin{array}{l}\mathrm{R}_{1}=\mathrm{R}_{2}=4 \text {-tert-butylphenyl, } \mathrm{X}=\mathrm{Et} \\
\mathrm{Y}=\mathrm{H}\end{array}$ & $\mathrm{R}_{1}-\mathrm{R}_{2}$ & 0.04 & - \\
\hline \multicolumn{4}{|l|}{ 9. Benzyl } \\
\hline a $\mathrm{R}_{1}=\mathrm{R}_{2}=$ phenyl, $\mathrm{R}_{3}=\mathrm{R}_{4}=\mathrm{R}_{5}=\mathrm{R}_{6}=\mathrm{H}$ & $\mathrm{R}_{1}-\mathrm{R}_{2}$ & 0.36 & 88 \\
\hline b $\mathrm{R}_{1}=\mathrm{R}_{2}=\mathrm{R}_{3}=$ phenyl, $\mathrm{R}_{4}=\mathrm{R}_{5}=\mathrm{R}_{6}=\mathrm{H}$ & $\mathrm{R}_{1}-\mathrm{R}_{2}=\mathrm{R}_{2}-\mathrm{R}_{3}$ & 0.20 & 21 \\
\hline c $\mathrm{R}_{2}=\mathrm{R}_{3}=\mathrm{R}_{5}=\mathrm{R}_{6}=$ phenyl, $\mathrm{R}_{1}=\mathrm{R}_{4}=\mathrm{H}$ & $R_{2}-R_{3}=R_{5}-R_{6}$ & 0.25 & $\mathrm{p}$ \\
\hline d $R_{1}=R_{2}=R_{3}=R_{4}=$ phenyl & $R_{1}-R_{2}=R_{3}-R_{4}$ & 0.175 & - \\
\hline $\mathrm{R}_{5}=\mathrm{R}_{6}=\mathrm{H}$ & $\mathrm{R}_{2}-\mathrm{R}_{3}$ & 0.162 & \\
\hline e $R_{1}=R_{2}=R_{3}=R_{4}=R_{5}=$ phenyl & $R_{1}-R_{2}=R_{4}-R_{5}$ & 0.149 & - \\
\hline $\mathrm{R}_{6}=\mathrm{H}$ & $R_{2}-R_{3}=R_{3}-R_{4}$ & 0.144 & \\
\hline
\end{tabular}

\begin{tabular}{|clll|}
\hline Table 2. (Continued) & & & \\
Molecules & & & \\
& Forming & $F^{*}{ }_{\pi, \sigma}\left(E_{h} /\right.$ & Yield \\
& ring & $\left.a_{0}\right)$ & {$[\%]$} \\
\hline$f \quad R_{1}=R_{2}=R_{3}=R_{4}=R_{5}=R_{6}=$ phenyl & $R_{1}-R_{2}$ & 0.125 & - \\
& $=R_{2}-R_{3}$ & & \\
& $=R_{3}-R_{4}$ & & \\
& $=R_{4}-R_{5}$ \\
& $=R_{5}-R_{6}$ \\
& $=R_{6}-R_{1}$ & & \\
& & \\
&
\end{tabular}
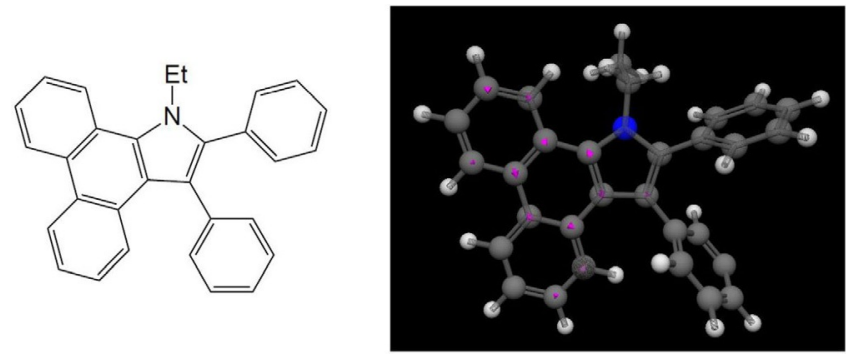

Figure 5. Molecular structure of $\mathrm{N}$-ethyl-2,3-diaryl-dibenzo[e,g]indole (left) and photo-induced atomic forces (right).

substituted and sterically crowded hexaphenylbenzene (9d) does not photocyclise while 1,2-diphenylbenzene (9a) and 1,2,3-triphenylbenzene $(\mathbf{9} \mathbf{b})$ do. This too can be explained by inspection of the photo-induced atomic forces for each molecule, as illustrated in Figure 6.

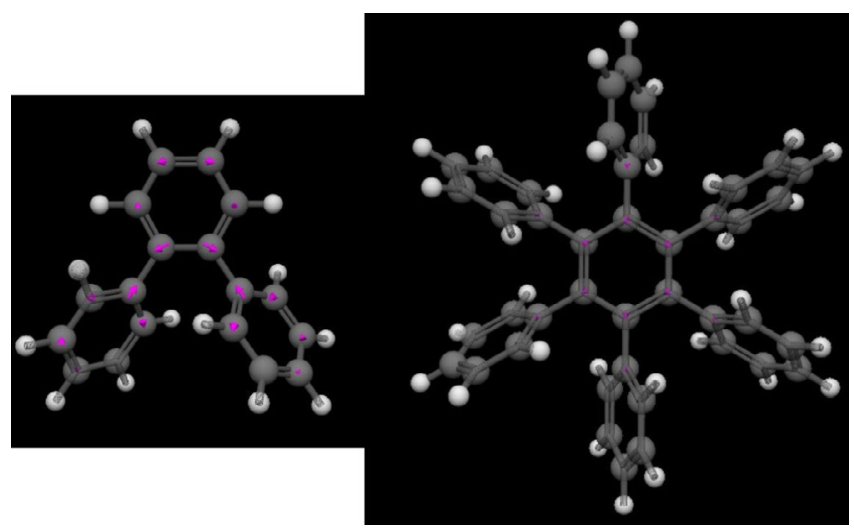

Figure 6. Photo-induced atomic forces within 1,2-diphenylbenzene (left) and hexaphenylbenzene (right).

In contradistinction to the dibenzo[e,g]indole case, bond aromatisation forces in hexaphenylbenzene are small not because the electronic transition is localised to an isolated and rigid molecular subfragment but rather because it is delocalised across the whole system.

Overall, there is a clear qualitative difference between molecules with strong bond aromatisation forces $\left(F_{\pi, \sigma}^{*}>0.15 E_{h} / a_{0}\right)$ that generally proceed to form planarised intermediates and photocyclised products, and those with weaker bond aromatisation forces $\left(\mathrm{F}_{\pi, \sigma}^{*}<0.15 \mathrm{E}_{\mathrm{h}} / \mathrm{a}_{0}\right)$ that do not. 
Therefore, we propose the following categorisation Scheme for predicting whether a polycyclic molecule will photoplanarise or not:

$\cdot \mathrm{F}_{\pi, \sigma}^{*}>0.15$-molecule will photoplanarise

$\cdot \mathrm{F}_{\pi, \sigma}^{*} \approx 0.15$-molecule may photoplanarise

$\cdot \mathrm{F}_{\pi, \sigma}^{*}<0.15$ molecule will not photoplanarise

Applying this categorization Scheme to our complete data set, we obtain the results shown in Table 3.

Table 3. Cross-correlation matrix of predicted planarisation against observed cyclisation for all molecules in our data set.

\begin{tabular}{|llll|} 
& & \multicolumn{2}{l}{ Observed cyclisation } \\
& & yes & no \\
\hline Predicted & yes & 29 & 0 \\
planarisation & no & 2 & 9 \\
\hline
\end{tabular}

Our model has high predictive power, with a strong correlation between predicted ability to photocyclise and observed product formation in experiments. Once again, this supports the hypothesis that the elimination step can be controlled by changing reaction conditions, but the overall outcome will be critically dependent on a molecule's intrinsic propensity to become aromatic in the excited state and hence undergo photoplanarisation.

However, there are two cases in which we predict that a molecule will not photoplanarise, yet it nonetheless is experimentally observed to photocyclise. Clearly our predictions are wrong, which warrants further more detailed investigation. Chemical structures and photo-induced atomic forces for 1phenylphenanthrene (2 e) and 1-(2-naphthyl)phenanthrene (2 f) are illustrated in Figure 7 and Figure 8, respectively. From these figures, it is firstly clear that these structures are closely related, comprising two rigid aromatic units connected by a

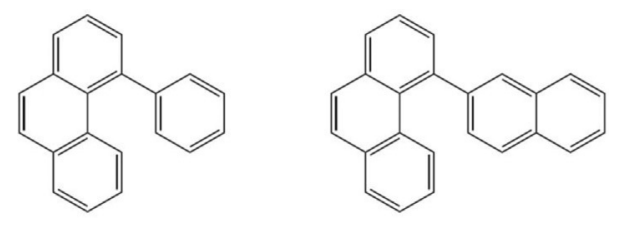

Figure 7. Molecular structure of 1-phenylphenanthrene (left) and 1-(2-naphthyl)phenanthrene (right).

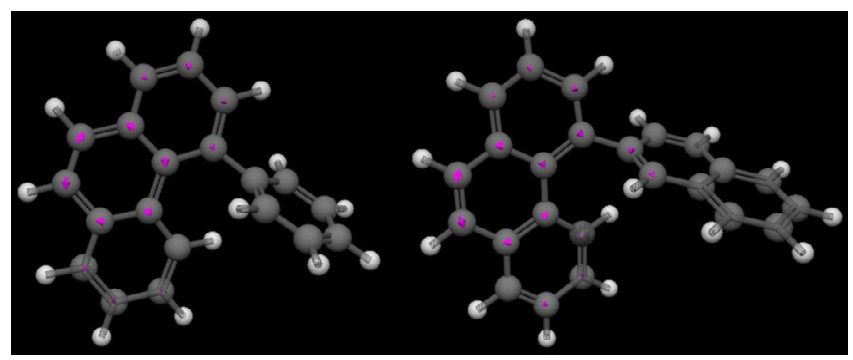

Figure 8. Photo-induced atomic forces within 1-phenylphenanthrene (left) and 1-(2-naphthyl)phenanthrene (right). single rotatable bond. In both cases, the cyclisation reaction occurs between the substituent and the scaffold. Close inspection of Figure 8 reveals that the photo-induced forces are predicted to largely localise on the phenanthrene unit, and do not correspond to aromatisation of the putative forming ring.

One possible explanation is that rotation about the single bond connecting the two aromatic units breaks $\pi$ conjugation and prevents aromatisation. However, the equilibrium torsion angles in these systems do not differ substantially from other molecules that are predicted to photoplanarise, which makes this explanation unlikely. Further, accounting for thermally accessible bond rotations would substantially increase the computational cost and complexity of our model, and prevent it from being routinely applied.

Alternatively, it is plausible that the electronic structure model we have chosen, TD-B3LYP/6-31G*, does not adequately model the excited state electronic structure of these particular systems. To test this hypothesis, we repeated our analysis using the Coulomb-attentuated version of B3LYP (CAM-B3LYP) functional designed to more accurately describe long-range interactions in electronically excited states. ${ }^{[55]}$ For both systems, we find that the excited state becomes more localised to the phenanthrene ring, and $F_{\pi, \sigma}^{*}$ drops to from 0.11 to $0.09 E_{h} / a_{0}$.

Overall, it is clear that photo-induced atomic forces computed at TD-B3LYP (with or without Coulomb attenuation) computed at the ground-state optimised geometry are not consistent with aromatisation of putative forming rings within these systems. This is a fundamental limitation of the approximations we have made in our model overall-in our choice of electronic structure model, and relatively simple computation and analysis procedure.

Nonetheless, we contend that our model still provides useful qualitative insights for very modest computational cost across a diverse set of molecules, even if it should be interpreted with caution for molecules of this type.

\subsection{Predicting preferred isomers}

Not only can our atomic force projection method predict whether photoplanarisation will occur or not, it can also predict the preferred photoplanarisation pathways if multiple options exist, that is, identify the pathway along which the largest total aromatisation forces will occur. Because the barrier to elimination is similar along all pathways, the favourability of the photoplanarisation step determines the reaction outcome.

In previous work, we have experimentally characterised the preferred constitutional isomer produced upon photocyclisation of $N$-ethyl-2,3,4,5-tetraphenylpyrrole $(\mathbf{6} \mathbf{b}) \cdot{ }^{[54]}$ Our current results show that the reaction pathway with the highest total aromatisation forces is the same pathway that leads to the experimentally observed product, as illustrated in Figure 9.

Similarly, 1,2-diphenyl-1,2-bis(thiophene-2-yl)ethene (1 f) has the potential to form two isomers, as represented in Figure 10. Again, we find that the pathway with the largest aromatisation force leads to the experimentally observed product.

On this basis, we are confident in predicting the outcome of previous reactions in which photocyclised products were ob- 


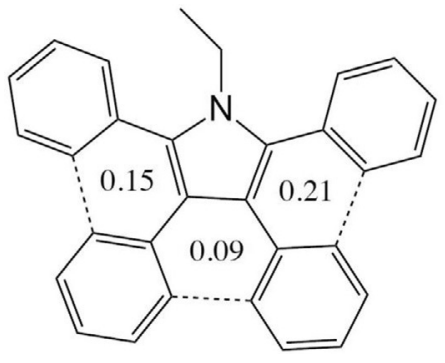

Figure 9. Total aromatisation forces for each putative forming ring within $\mathrm{N}$ ethyl-2,3,4,5-tetraphenylpyrrole (6 b).

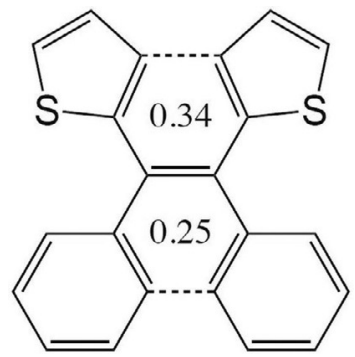

Figure 10. Total aromatisation forces for each putative forming ring within 1,2-diphenyl-1,2-bis(thiophene-2-yl)ethene (1 f).

served but not resolved down to the individual-isomer level for molecules 2,3,4,5-tetraphenylfuran (4b), 2,3,4,5-tetraphenylthiophene (5 b) and 2,3,4,5-tetraphenylpyrrole (6a), based upon the results presented in Table 2 . In all cases, we predict photocyclisation between the phenyl rings at positions 2 and 3. This prediction would be interesting to confirm experimentally.

\subsection{Synthetic implications}

A molecule's propensity to photoplanarise is determined by the nature of its UV electronic excitation. To promote aromatisation, this transition must primarily and substantially alter the electronic structure within the putative forming ring. This cannot be achieved if the electronic transition is localised on a rigid subfragment, nor if the transition is delocalised over the entire molecule.

Molecular characteristics that promote planarisation are therefore:

-Asymmetry in molecular structure and/or charge distribution, including
- Inclusion of heteroaromatic ring substituents and ethylene bridges

-A limited number of potential photocyclisation sites and/or structurally well-separated sites

-Absence of large aromatic subunits upon which localised excited state formation is likely, or structural distortion to disrupt their aromaticity (e.g. helicenes)

Within our data set, we have included molecules that correspond to the photocyclised products of other, less conjugated reactant molecules in the same set. For example, the dibenzo[e,g]indoles are photocyclisation products of the pyrroles, and some phenanthrenes are photocyclisation products of the stilbenes. These reaction pathways are illustrated in Figure 11 and Figure 12.

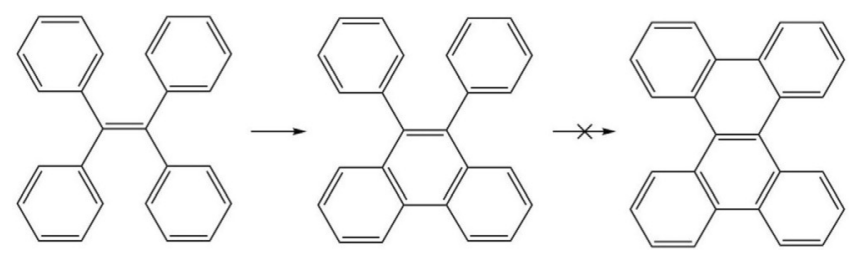

Figure 11. Consecutive potential photocyclisation pathways for $1,1^{\prime}, 2,2^{\prime}$-tetraphenylethene.

We uniformly find that the second photoplanarisation reaction in each sequence is less facile than the first. In both cases studied here, the total bond aromatisation force during the second reaction in insufficient to drive the photoplanarisation process to completion and form the pseudo-aromatic intermediate. Overall, our results suggest that photocyclisation reactions cannot readily be used to form large, fused polyaromatic ring systems through a process of sequential cyclisation reactions to a growing aromatic core. However, alternative strategies are possible.

In the pioneering work of Laarhoven et al., ${ }^{[36,37]}$ extended polyaromatic systems were synthesised by tethering two polyaromatic systems with an ethylene bridging linker, and then photocyclising around this stilbene-like core. More recently, it has been shown that including highly polar lactones within the polyaromatic core promotes photocyclisation, particularly if they are placed adjacent to the photocyclisation centres. ${ }^{[56]}$ This strategy involves incorporating molecular characteristics that promote planarisation (inclusion of heteroatoms) to overcome those that would otherwise be detrimental (large existing aromatic core).

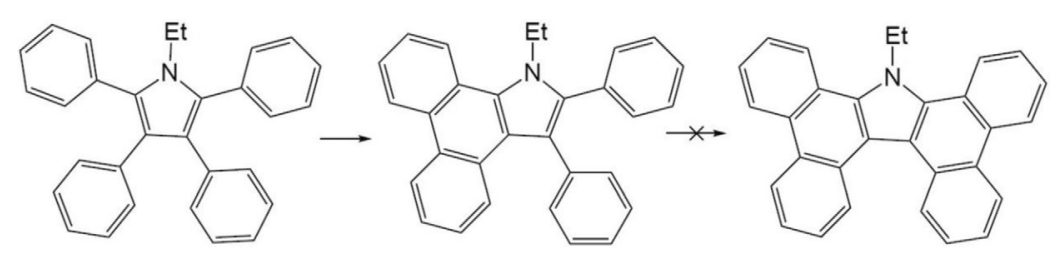

Figure 12. Consecutive potential photocyclisation pathways for 2,3,4,5-tetraphenylpyrrole. 


\section{Conclusion}

We have introduced a universally applicable ab initio computational tool for predicting the intrinsic ability of a molecule to aromatise and planarise following photo-excitation, and applied it to predict and rationalise the outcome of a series of known and newly characterised photocyclisation reactions. We find a strong correlation between a molecule's propensity to photoplanarise and whether the photocyclisation reaction proceeds to completion experimentally. This implies that photoplanarisation is the primary reaction-determining step in the overall photocyclisation process, although the elimination step may still be rate determining, depending on the reaction conditions.

\section{Experimental Methods}

Unless otherwise specified, all reagents and starting materials were reagent grade, purchased from standard suppliers and used as received. Melting points were recorded on an electrothermal melting point apparatus and are uncorrected. Elemental analysis was carried out by Campbell Microanalytical Laboratory, University of Otago. All infrared spectra were recorded on a PerkinElmer Spectrum One FTIR instrument. Mass spectra were on a DIONEX Ultimate 3000 or Bruker MaXis 4G spectrometer, both of which were operated in high resolution positive ion electrospray mode. All spectra were recorded on a Varian INOVA 500, Varian Unity 300, or an Agilent 400-MR instrument operating at 500,300 and $400 \mathrm{MHz}$, respectively, for ${ }^{1} \mathrm{H}$, and 125,75 and $125 \mathrm{MHz}$, respectively, for ${ }^{13} \mathrm{C}$. Spectra were referenced to the residual solvent peaks and/or TMS. COSY, HSQC and HMBC experiments were employed where required, using standard Varian and Agilent pulse sequences. UV/Visible spectra were recorded on a Varian CARY UV/Visible spectrometer. Emission spectra were recorded on a Horiba Fluorolog-3. See ESI for details of the synthesis of $7 a-7 h$.

\section{General procedure for the synthesis of dibenzo[a,c]carba- zoles using photocyclisation}

In an oven dry quartz tube flushed with argon, $400 \mathrm{~mL}$ of dry toluene was added, and sparged with argon for 5 minutes. The appropriate indole was added, along with $I_{2}$ and propylene oxide. The solution was sparged with argon for 15 minutes and then irradiated with $300 \mathrm{~nm}$ light in a Rayonet photoreactor overnight with argon bubbling through the solution continuously. The solution was then washed with $200 \mathrm{~mL} \mathrm{Na} \mathrm{S}_{2} \mathrm{O}_{3}$ solution and $100 \mathrm{~mL} \mathrm{H} \mathrm{H}_{2} \mathrm{O}$, dried with $\mathrm{MgSO}_{4}$ and the solvent removed in vacuo. The residue was purified by flash column chromatography.

\section{Synthesis of 9-ethyl-9H-dibenzo[ $[a, c]$ carbazole, $10 \mathrm{a}$}

7 a $(0.150 \mathrm{~g}, 0.504 \mathrm{mmol}), \mathrm{l}_{2}(0.140 \mathrm{~g}, 5.55 \mathrm{mmol})$, propylene oxide $(1.5 \mathrm{~mL})$, purification with flash column chromatography $\left(\mathrm{SiO}_{2} 3: 2\right.$ hexanes:dichloromethane). Crystals suitable for X-ray crystallography were obtained by recrystallisation from ethyl acetate and hexanes. Yield: $0.095 \mathrm{~g}$ (64\%). m.p. $114-118^{\circ} \mathrm{C}$; ${ }^{1} \mathrm{H}$ NMR $(400 \mathrm{MHz}$, $\left.\mathrm{CDCl}_{3}\right): \delta_{\mathrm{H}}=8.90(\mathrm{~d}, 2 \mathrm{H}, J=8.6 \mathrm{~Hz}), 8.79(\mathrm{~d}, 1 \mathrm{H}, J=8.6 \mathrm{~Hz}), 8.65(\mathrm{~d}$, $1 \mathrm{H}, J=8 \mathrm{~Hz}), 8.56(\mathrm{~d}, 1 \mathrm{H}, J=7.4 \mathrm{~Hz}), 7.77-7.69(\mathrm{~m}, 3 \mathrm{H}), 7.67(\mathrm{~d}$, $1 \mathrm{H}, J=8 \mathrm{~Hz}), 7.59(\mathrm{t}, 1 \mathrm{H}, J=7.4 \mathrm{~Hz}), 7.51(\mathrm{t}, 1 \mathrm{H}, J=8 \mathrm{~Hz}), 7.42(\mathrm{t}$, $1 \mathrm{H}, J=8 \mathrm{~Hz}), 4.90\left(\mathrm{q}, 2 \mathrm{H}, \mathrm{CH}_{2}, J=7 \mathrm{~Hz}, \mathrm{H} 13\right), 1.74 \mathrm{ppm}\left(\mathrm{t}, 3 \mathrm{H}, \mathrm{CH}_{3}\right.$, $J=7 \mathrm{~Hz}, \mathrm{H} 14) ;{ }^{13} \mathrm{C}$ NMR $\left(100 \mathrm{MHz}, \mathrm{CDCl}_{3}\right): \delta_{\mathrm{C}}=140.0,133.6,130.9$, 130.0, 127.3, 126.8, 126.4, 125.5, 124.2, 123.7, 123.6, 123.5, 123.4,
123.3, 122.5, 121.9, 120.3, 113.6, 109.4, 40.9, 15.3 ppm; ESMS: calc for $\mathrm{C}_{22} \mathrm{H}_{18} \mathrm{~N}\left([2.15+\mathrm{H}]^{+}\right)=296.1434$, found 296.1428, calc for $\mathrm{C}_{22} \mathrm{H}_{17} \mathrm{~N}\left([2.15 \mathrm{M}]^{+}\right)=295.1356$, found 295.1358; IR (KBr): $\tilde{v}=2969$ $(\mathrm{m}), 1608(\mathrm{~m}), 1516(\mathrm{~s}), 1467(\mathrm{~s}), 1444(\mathrm{~s}), 1371(\mathrm{~s}), 1350(\mathrm{~s}), 1224$ $(\mathrm{m}), 1156(\mathrm{~m}), 1094(\mathrm{~m}), 935(\mathrm{~m}), 745(\mathrm{~s}), 733(\mathrm{~s}), 716 \mathrm{~cm}^{-1}(\mathrm{~s})$; UV/ Vis $\lambda_{\max }(\varepsilon): 275 \mathrm{~nm}$ (52363), $297 \mathrm{~nm}$ (15472), $326 \mathrm{~nm}$ (17219), $359 \mathrm{~nm}$ (4877), $378 \mathrm{~nm}$ (4659); Fluorometry $\lambda_{\max }: 389 \mathrm{~nm}, 407 \mathrm{~nm}$.

\section{Synthesis of 9-ethyl-3,6-dimethoxy-9H-dibenzo[a,c]carba- zole, $10 \mathrm{~b}$}

$7 \mathrm{~b}(0.150 \mathrm{~g}, 0.420 \mathrm{mmol}), \mathrm{l}_{2}(0.120 \mathrm{~g}, 0.462 \mathrm{mmol})$, propylene oxide $(1.5 \mathrm{~mL})$, purification with flash column chromatography $\left(\mathrm{SiO}_{2}\right.$, dichloromethane). Yield: $0.090 \mathrm{~g}$ (60\%). m.p. $96-99^{\circ} \mathrm{C}$; ${ }^{1} \mathrm{H}$ NMR $\left(400 \mathrm{MHz}, \mathrm{CDCl}_{3}\right): \delta_{\mathrm{H}}=8.76(\mathrm{~d}, 1 \mathrm{H}, J=9 \mathrm{~Hz}, \mathrm{H} 1), 8.54(\mathrm{~d}, 1 \mathrm{H}, J=$ $8 \mathrm{~Hz}, \mathrm{H} 7), 8.37(\mathrm{~d}, 1 \mathrm{H}, J=9 \mathrm{~Hz}, \mathrm{H} 2), 8.11(\mathrm{~s}, 1 \mathrm{H}, \mathrm{H} 3), 8.04(\mathrm{~s}, 1 \mathrm{H}$, $\mathrm{H} 5), 7.57(\mathrm{~d}, 1 \mathrm{H}, J=8 \mathrm{~Hz}, \mathrm{H} 9), 7.48(\mathrm{t}, 1 \mathrm{H}, J=7.5 \mathrm{~Hz}, \mathrm{H} 10), 7.40(\mathrm{~d}$, $1 \mathrm{H}, J=8 \mathrm{~Hz}, \mathrm{H6}), 7.29(\mathrm{t}, 1 \mathrm{H}, J=7.5 \mathrm{~Hz}, \mathrm{H} 11), 7.21(\mathrm{~d}, 1 \mathrm{H}, J=$ $7.5 \mathrm{~Hz}, \mathrm{H} 12), 4.72\left(\mathrm{q}, 2 \mathrm{H}, \mathrm{CH}_{2}, J=7 \mathrm{~Hz}, \mathrm{H} 13\right), 4.03\left(\mathrm{~s}, 6 \mathrm{H}, \mathrm{OCH}_{3}, \mathrm{H} 4\right.$, $\mathrm{H} 8), 1.66 \mathrm{ppm}(\mathrm{t}, 3 \mathrm{H}, \mathrm{CH} 3, J=7 \mathrm{~Hz}, \mathrm{H} 13) ;{ }^{13} \mathrm{CNMR}(100 \mathrm{MHz}$, $\left.\mathrm{CDCl}_{3}\right): \delta_{\mathrm{C}}=157.2,155.9,139.87,132.8,132.2,127.7,124.9,124.1$, $123.5,123.3,121.4,120.1,118.4,116.3,115.5,112.1,109.2,106.8$, 106.3, 55.6, 55.5, 40.9, 15.2 ppm; ESMS: calc for $\mathrm{C}_{24} \mathrm{H}_{22} \mathrm{NO}_{2}$ ([2.16+ $\left.\mathrm{H}]^{+}\right)=356.1645$, found 356.1630, calc for $\mathrm{C}_{24} \mathrm{H}_{21} \mathrm{NO}_{2}\left([2.16 \mathrm{M}]^{+}\right)=$ 355.1567, found 355.167; IR (KBr): $\tilde{v}=2983(\mathrm{~m}), 2929(\mathrm{~m}), 1616(\mathrm{~m})$, $1574(\mathrm{~m}), 1528(\mathrm{~m}), 1465(\mathrm{~s}), 1377(\mathrm{~m}), 1236(\mathrm{~s}), 1038(\mathrm{~m}), 833(\mathrm{~m})$, $800(\mathrm{~m}), 789(\mathrm{~m}), 737 \mathrm{~cm}^{-1}(\mathrm{~m}) ;$ UV/Vis $\lambda_{\max }(\varepsilon): 277 \mathrm{~nm}(59286)$, $310 \mathrm{~nm}$ (23439), $329 \mathrm{~nm}$ (18347), $380 \mathrm{~nm}$ (4693), $400 \mathrm{~nm}$ (5080); Fluorometry $\lambda_{\max }: 420 \mathrm{~nm}, 435 \mathrm{~nm}$.

\section{Synthesis of 9-ethyl-3,6-dimethyl-9H-dibenzo[a,c]carbazole, $10 \mathrm{c}$}

$7 \mathrm{c}(0.150 \mathrm{~g}, 0.461 \mathrm{mmol}), \mathrm{l}_{2}(0.130 \mathrm{~g}, 0.507 \mathrm{mmol})$, propylene oxide $(1.5 \mathrm{~mL})$, purification with flash column chromatography $\left(\mathrm{SiO}_{2}, 3: 2\right.$ hexanes:dichloromethane). Crystals suitable for X-ray crystallography were obtained by recrystallisation from ethyl acetate and hexanes. Yield: $0.130 \mathrm{~g}$ (85\%). m.p. $137-141^{\circ} \mathrm{C} ;{ }^{1} \mathrm{H}$ NMR $(400 \mathrm{MHz}$, $\left.\mathrm{CDCl}_{3}\right): \delta_{\mathrm{H}}=8.77(\mathrm{~d}, 1 \mathrm{H}, J=8.4 \mathrm{~Hz}, \mathrm{H} 1), 8.67(\mathrm{~s}, 1 \mathrm{H}, \mathrm{H} 3), 8.60(\mathrm{~d}$, $1 \mathrm{H}, J=8 \mathrm{~Hz}, \mathrm{H} 9), 8.57(\mathrm{~s}, 1 \mathrm{H}, \mathrm{H} 5), 8.45(\mathrm{~d}, 1 \mathrm{H}, J=8.4 \mathrm{~Hz}, \mathrm{H} 7), 7.63$ $(\mathrm{d}, 1 \mathrm{H}, J=8.4 \mathrm{~Hz}, \mathrm{H} 2), 7.57(\mathrm{~d}, 1 \mathrm{H}, J=8.4 \mathrm{~Hz}, \mathrm{H6}), 7.53(\mathrm{~d}, 1 \mathrm{H}, J=$ $8 \mathrm{~Hz}, \mathrm{H} 12), 7.49(\mathrm{t}, 1 \mathrm{H}, J=8 \mathrm{~Hz}, \mathrm{H} 11), 7.39(\mathrm{t}, 1 \mathrm{H}, J=8 \mathrm{~Hz}, \mathrm{H} 10)$, 4.87 (q, $\left.2 \mathrm{H}, \mathrm{CH}_{2}, \mathrm{H} 13\right), 2.67\left(\mathrm{~s}, 3 \mathrm{H}, \mathrm{CH}_{3}, \mathrm{H} 4\right), 2.66\left(\mathrm{~s}, 3 \mathrm{H}, \mathrm{CH}_{3}, \mathrm{H} 8\right)$, $1.72 \mathrm{ppm}\left(\mathrm{t}, 3 \mathrm{H}, \mathrm{CH}_{3}, \mathrm{H} 14\right) ;{ }^{13} \mathrm{C}$ NMR $\left(100 \mathrm{MHz}, \mathrm{CDCl}_{3}\right): \delta_{\mathrm{C}}=140.0$, 135.0, 133.4, 132.7, 130.9, 128.7, 127.9, 127.8, 126.8, 124.1, 123.7, 123.4, 123.3, 123.2, 122.4, 121.8, 121.5, 120.1, 113.0, 109.3, 40.8, 21.9, 21.8, 15.3 ppm; ESMS: calc for $\mathrm{C}_{24} \mathrm{H}_{22} \mathrm{~N}\left([2.17+\mathrm{H}]^{+}\right)=$ 324.1747, found 324.1733, calc for $\mathrm{C} 24 \mathrm{H} 21 \mathrm{H}\left([2.17 \mathrm{M}]^{+}\right)=323.1669$, found 323.1668; IR (KBr): $\tilde{v}=2975(\mathrm{w}), 2919(\mathrm{w}), 1528(\mathrm{~s}), 1466(\mathrm{~s})$, $1371(\mathrm{~m}), 1348(\mathrm{~m}), 1333(\mathrm{~m}), 1297(\mathrm{~m}), 1226(\mathrm{~m}), 802(\mathrm{~s}), 739 \mathrm{~cm}^{-1}$ (s); UV/Vis $\lambda_{\max }(\varepsilon): 277 \mathrm{~nm}$ (61425), $302 \mathrm{~nm} \quad(19026), 327 \mathrm{~nm}$ (19612), $365 \mathrm{~nm}$ (5226), $384 \mathrm{~nm}$ (5212); Fluorometry $\lambda_{\max }: 397 \mathrm{~nm}$, $414 \mathrm{~nm}$.

\section{Synthesis of 9-ethyl-3,6-ditertbutyl-9H-dibenzo[a,c]carba- zole, $10 \mathrm{~d}$}

$7 \mathrm{~d}(0.150 \mathrm{~g}, 0.366 \mathrm{mmol}), \mathrm{l}_{2}(0.100 \mathrm{~g}, 0.403 \mathrm{mmol})$, propylene oxide $(1.5 \mathrm{~mL})$, purification with flash column chromatography $\left(\mathrm{SiO}_{2}, 3: 2\right.$ hexanes:dichloromethane). Yield: $0.133 \mathrm{~g}(89 \%)$. m.p. $135-138^{\circ} \mathrm{C}$; ${ }^{1} \mathrm{H}$ NMR $\left(400 \mathrm{MHz}, \mathrm{CDCl}_{3}\right): \delta_{\mathrm{H}}=8.92(\mathrm{~s}, 1 \mathrm{H}, \mathrm{H} 3), 8.83(\mathrm{~d}, 1 \mathrm{H}, J=$ $8.8 \mathrm{~Hz}, \mathrm{H} 1), 8.81(\mathrm{~s}, 1 \mathrm{H}, \mathrm{H} 5), 8.62(\mathrm{~d}, 1 \mathrm{H}, J=7.6 \mathrm{~Hz}, \mathrm{H9}), 8.51(\mathrm{~d}, 1 \mathrm{H}$, $J=8.8 \mathrm{~Hz}, \mathrm{H} 2), 7.84(\mathrm{~d}, 1 \mathrm{H}, J=8.4 \mathrm{~Hz}, \mathrm{H} 7), 7.79(\mathrm{~d}, 1 \mathrm{H}, J=8.4 \mathrm{~Hz}$, H6), $7.63(\mathrm{~d}, 1 \mathrm{H}, J=7.6 \mathrm{~Hz}, \mathrm{H} 12), 7.50(\mathrm{t}, 1 \mathrm{H}, J=7.6 \mathrm{~Hz}, \mathrm{H} 11), 7.41$ 
$(\mathrm{t}, 1 \mathrm{H}, J=7.6 \mathrm{~Hz}, \mathrm{H} 10), 4.87\left(\mathrm{q}, 2 \mathrm{H}, \mathrm{CH}_{2}, J=7 \mathrm{~Hz}, \mathrm{H} 13\right), 1.73(\mathrm{t}, 1 \mathrm{H}$, $\left.\mathrm{CH}_{3}, J=7 \mathrm{~Hz}, \mathrm{H} 14\right), 1.56 \mathrm{ppm}\left(\mathrm{s}, 18 \mathrm{H}, \mathrm{CH}_{3}, \mathrm{H} 4, \mathrm{H} 8\right) ;{ }^{13} \mathrm{C} N M R$ $\left(100 \mathrm{MHz}, \mathrm{CDCl}_{3}\right): \delta_{\mathrm{C}}=148.0,145.8,139.9,133.5,130.8,127.9,126.6$, $125.3,124.4,123.7,123.4,123.3,122.4,121.8,121.5,120.1,119.8$, 118.9, 113.0, 109.3, 40.9, 31.5, 31.4, 15.3 ppm; ESMS: calc for $\mathrm{C}_{30} \mathrm{H}_{34} \mathrm{~N}\left([2.18+\mathrm{H}]^{+}\right)=408.2686$, found 408.2684; IR (KBr): $\tilde{v}=2956$ (s), $2902(\mathrm{~m}, \mathrm{sh}), 2866(\mathrm{~m}, \mathrm{sh}), 1525(\mathrm{~m}), 1463(\mathrm{~s}), 1372(\mathrm{~m}), 1361(\mathrm{~m})$, $1344(\mathrm{~m}), 1333(\mathrm{~m}), 1260(\mathrm{~m}), 879(\mathrm{~m}), 814(\mathrm{~m}), 733(\mathrm{~s}), 602 \mathrm{~cm}^{-1}$ $\left.(\mathrm{m}) ; \quad U V / V i s \lambda_{\max }(\varepsilon)\right): 277 \mathrm{~nm}(63644), 299 \mathrm{~nm}(18444), 327 \mathrm{~nm}$ (19505), $362 \mathrm{~nm}$ (5523), $381 \mathrm{~nm}$ (5688); Fluorometry $\lambda_{\max }: 392 \mathrm{~nm}$, $410 \mathrm{~nm}$.

\section{Synthesis of 9-benzyl-9H-dibenzo[a,c]carbazole, $10 \mathrm{e}$}

7 e $(0.150 \mathrm{~g}, 0.417 \mathrm{mmol}), \mathrm{I}_{2}(0.120 \mathrm{~g}, 0.459 \mathrm{mmol})$, propylene oxide $(1.5 \mathrm{~mL})$, purification with flash column chromatography $\left(\mathrm{SiO}_{2}, 3: 2\right.$ hexanes:dichloromethane). Yield: $0.125 \mathrm{~g}$ (84\%). m.p. $141-143^{\circ} \mathrm{C}$; ${ }^{1} \mathrm{H}$ NMR $\left(400 \mathrm{MHz}, \mathrm{CDCl}_{3}\right): \delta_{\mathrm{H}}=8.94(\mathrm{~d}, 1 \mathrm{H}, J=7.6 \mathrm{~Hz}), 8.86(\mathrm{~d}, 1 \mathrm{H}$, $J=8.4 \mathrm{~Hz}), 8.80(\mathrm{~d}, 1 \mathrm{H}, J=7.6 \mathrm{~Hz}), 8.70(\mathrm{~s}, 1 \mathrm{H}, J=8.4 \mathrm{~Hz}), 8.27(\mathrm{~d}$, $1 \mathrm{H}, J=8.4 \mathrm{~Hz}), 7.791(\mathrm{t}, 1 \mathrm{H}, J=7.6 \mathrm{~Hz}), 7.63-7.61(\mathrm{~m}, 2 \mathrm{H}), 7.51-$ $7.44(\mathrm{~m}, 4 \mathrm{H}), 7.38-7.32(\mathrm{~m}, 5 \mathrm{H}), 6.03 \mathrm{ppm}\left(\mathrm{s}, 2 \mathrm{H}, \mathrm{CH}_{2}, \mathrm{H} 13\right)$; ${ }^{13} \mathrm{C} \mathrm{NMR}\left(100 \mathrm{MHz}, \mathrm{CDCl}_{3}\right): \delta_{\mathrm{C}}=141.2,137.4,134.7,130.9,129.9$, $129.1,127.5,127.4,127.0,126.5,126.0,125.7,124.1,124.0,123.8$, 123.7, 123.5, 123.2, 122.9, 121.9, 120.9, 110.0, 50.2 ppm; ESMS: calc for $\mathrm{C}_{27} \mathrm{H}_{20} \mathrm{~N}\left([2.19+\mathrm{H}]^{+}\right)=358.1590$, found 358.1594; IR (KBr): $\tilde{v}=$ $3041(\mathrm{w}), 1514(\mathrm{~m}), 1470(\mathrm{~s}), 1359(\mathrm{~m}), 1334(\mathrm{~m}), 1210(\mathrm{w}), 1155$ (w), $746(\mathrm{~s}), 729(\mathrm{~m}), 719 \mathrm{~cm}^{-1}(\mathrm{~m})$; UV/Vis $\lambda_{\max }(\varepsilon): 274 \mathrm{~nm}(56103)$, $295 \mathrm{~nm}$ (16745), $324 \mathrm{~nm}$ (19190), $357 \mathrm{~nm}$ (5568), $375 \mathrm{~nm} \mathrm{(5247);}$ Fluorometry $\lambda_{\max }: 385 \mathrm{~nm}, 402 \mathrm{~nm}$.

\section{Synthesis of 9-benzyl-3,6-dimethoxy-9H-dibenzo[a,c]carba- zole, $10 \mathrm{f}$}

$7 \mathrm{f}(0.150 \mathrm{~g}, 0.358 \mathrm{mmol}), \mathrm{FeCl}_{3}(0.87 \mathrm{~g}, 5.36 \mathrm{mmol})$, the dark red residue was purified by flash chromatography $\left(\mathrm{SiO}_{2}, 4: 1\right.$ hexanes:ethyl acetate). Crystals suitable for X-ray crystallography were obtained by slow diffusion of diisopropyl ether into a benzene solution of 2.20. Yield: $0.035 \mathrm{~g}$ (23\%). m.p. $191-194^{\circ} \mathrm{C}$; ${ }^{1} \mathrm{H}$ NMR $\left(400 \mathrm{MHz}, \mathrm{CDCl}_{3}\right): \delta_{\mathrm{H}}=8.84(\mathrm{~d}, \mathrm{br}, 1 \mathrm{H}, \mathrm{H} 1), 8.59(\mathrm{~d}, \mathrm{br}, 1 \mathrm{H}, \mathrm{H} 9), 8.17$ (d, $1 \mathrm{H}, J=8.4 \mathrm{~Hz}, \mathrm{H} 7), 8.15(\mathrm{~s}, 1 \mathrm{H}, \mathrm{H} 3), 8.10(\mathrm{~s}, 1 \mathrm{H}, \mathrm{H} 5), 7.45-7.30$ $(\mathrm{m}, 9 \mathrm{H}, \mathrm{H} 2, \mathrm{H} 10), 7.12(\mathrm{~d}, 1 \mathrm{H}, J=8.4 \mathrm{~Hz}, \mathrm{H} 6), 5.96\left(\mathrm{~s}, 2 \mathrm{H}, \mathrm{CH}_{2}, \mathrm{H} 13\right)$, $4.06\left(\mathrm{~s}, 3 \mathrm{H}, \mathrm{OCH}_{3}, \mathrm{H} 8\right), 4.00 \mathrm{ppm}(\mathrm{s}, 3 \mathrm{H}, \mathrm{OCH} 3, \mathrm{H} 4) ;{ }^{13} \mathrm{C} N M R$ $\left(125 \mathrm{MHz}, \mathrm{CDCl}_{3}\right): \delta_{\mathrm{C}}=157.4,137.6,132.2,129.1,127.5,126.0,125.1$ C1, 124.5 C7, 123.6, 121.4 C9, 120.6, 116.5 C6, 115.3, 109.7, 106.9 C3, 106.4 C5, $55.6 \mathrm{C} 8,55.4 \mathrm{C} 4,50.2 \mathrm{ppm}$ C13; ESMS: calc for $\mathrm{C}_{29} \mathrm{H}_{24} \mathrm{NO}_{2} \quad\left([2.20+\mathrm{H}]^{+}\right)=418.1802$, found 418.1786 ; calc for $\mathrm{C}_{29} \mathrm{H}_{23} \mathrm{NO}_{2}\left([2.20 \mathrm{M}]^{+}\right)=417.1723$, found, 417.1726; IR (KBr): $\tilde{v}=2953$ (w), $2927(\mathrm{w}), 1616(\mathrm{~m}), 1529(\mathrm{~m}), 1469(\mathrm{~s}), 1238(\mathrm{~s}), 1207(\mathrm{~s}), 1038$ (s), $836(\mathrm{~s}), 803(\mathrm{~s}), 732(\mathrm{~s}), 702 \mathrm{~cm}^{-1}(\mathrm{~m}) ;$ UV/Vis $\lambda_{\max }(\varepsilon): 276 \mathrm{~nm}$ (49464), $309 \mathrm{~nm}$ (19528), $327 \mathrm{~nm}$ (16030), $378 \mathrm{~nm}$ (3900), $398 \mathrm{~nm}$ (4416); Fluorometry $\lambda_{\text {max }}: 415 \mathrm{~nm}, 431 \mathrm{~nm}$.

\section{Synthesis of 9-benzyl-3,6-dimethyl-9H-dibenzo[a,c]carba- zole, $10 \mathrm{~g}$}

$7 \mathrm{~g}(0.150 \mathrm{~g}, 0.387 \mathrm{mmol}), \mathrm{l}_{2}(0.110 \mathrm{~g}, 0.426 \mathrm{mmol})$, propylene oxide $(1.5 \mathrm{~mL})$, purification with flash column chromatography $\left(\mathrm{SiO}_{2}, 3: 2\right.$ hexanes:dichloromethane). Crystals suitable for X-ray crystallography were obtained by recrystallisation from ethyl acetate and hexanes. Yield: $0.084 \mathrm{~g}$ (56\%). m.p. $216-218^{\circ} \mathrm{C}$; ${ }^{1} \mathrm{H}$ NMR $(400 \mathrm{MHz}$, $\left.\mathrm{CDCl}_{3}\right): \delta_{\mathrm{H}}=8.81(\mathrm{~d}, 1 \mathrm{H}, J=8.4 \mathrm{~Hz}, \mathrm{H} 1), 8.63(\mathrm{~s}, 1 \mathrm{H}, \mathrm{H} 3), 8.57(\mathrm{~s}, 1 \mathrm{H}$, $\mathrm{H} 5), 8.16(\mathrm{~d}, 1 \mathrm{H}, J=7.6 \mathrm{~Hz}, \mathrm{H} 6), 7.60(\mathrm{~d}, 1 \mathrm{H}, J=8.4 \mathrm{~Hz}, \mathrm{H} 12), 7.47-$ $7.29(\mathrm{~m}, 10 \mathrm{H}), 6.00\left(\mathrm{~s}, 2 \mathrm{H}, \mathrm{CH}_{2}, \mathrm{H} 13\right), 2.67\left(\mathrm{~s}, 3 \mathrm{H}, \mathrm{CH}_{3}, \mathrm{H} 4\right)$, $2.60 \mathrm{ppm}\left(\mathrm{s}, 3 \mathrm{H}, \mathrm{CH}_{3}, \mathrm{H} 8\right) ;{ }^{13} \mathrm{C} \mathrm{NMR}\left(100 \mathrm{MHz}, \mathrm{CDCl}_{3}\right)$ : $\delta_{\mathrm{C}}=141.7$,
137.6, 135.2, 134.8, 133.0, 131.2, 129.1, 128.9, 127.9, 127.8, 127.5, $127.1,126.0,124.0,123.8,123.5,123.4,122.8,121.8,121.1,120.6$, 109.8, 50.1, 22.0, 21.9 ppm; ESMS: calc for $\mathrm{C}_{29} \mathrm{H}_{24} \mathrm{~N}\left([2.21+\mathrm{H}]^{+}\right)=$ 386.1904, found 386.1873, calc for $\mathrm{C}_{29} \mathrm{H}_{23} \mathrm{~N}\left([2.21 \mathrm{M}]^{+}\right)=385.1825$, found 385.1828; IR (KBr): $\tilde{v}=2917(\mathrm{w}, \mathrm{br}), 1528(\mathrm{~m}), 1467(\mathrm{~m}), 1454$ $(\mathrm{m}), 1357(\mathrm{~m}), 1332(\mathrm{~m}), 803(\mathrm{~s}), 735(\mathrm{~s}), 696 \mathrm{~cm}^{-1}(\mathrm{w})$; UV/Vis $\lambda_{\max }$ $(\varepsilon): 276 \mathrm{~nm}$ (65698), $300 \mathrm{~nm}$ (19672), $326 \mathrm{~nm}$ (21264), $363 \mathrm{~nm}$ (5463), $382 \mathrm{~nm}$ (5570); Fluorometry $\lambda_{\max }: 394 \mathrm{~nm}, 411 \mathrm{~nm}$.

\section{Synthesis of 9-benzyl-3,6-ditertbutyl-9H-dibenzo[ $a, c]$ carba- zole, $10 \mathrm{~h}$}

$7 \mathrm{~h}(0.150 \mathrm{~g}, 0.318 \mathrm{mmol}), \mathrm{I}_{2}(0.090 \mathrm{~g}, 0.350 \mathrm{mmol})$, propylene oxide $(1.5 \mathrm{~mL})$, purification with flash column chromatography $\left(\mathrm{SiO}_{2}, 7: 3\right.$ hexanes:dichloromethane). Yield: $0.120 \mathrm{~g}$ (78\%). m.p. $179-182^{\circ} \mathrm{C}$; ${ }^{1} \mathrm{H}$ NMR $\left(400 \mathrm{MHz}, \mathrm{CDCl}_{3}\right): \delta_{\mathrm{H}}=8.87(\mathrm{~s}, 1 \mathrm{H}, \mathrm{H} 3), 8.85(\mathrm{~d}, 1 \mathrm{H}, J=$ $7.6 \mathrm{~Hz}, \mathrm{H} 2), 8.80(\mathrm{~s}, 1 \mathrm{H}, \mathrm{H} 5), 8.66(\mathrm{~d}, 1 \mathrm{H}, J=8.8 \mathrm{~Hz}, \mathrm{H} 6), 8.21(\mathrm{~d}, 1 \mathrm{H}$, $J=8.8 \mathrm{~Hz}), 7.85(\mathrm{~d}, 1 \mathrm{H}, J=8.8 \mathrm{~Hz}), 7.54(\mathrm{~d}, 1 \mathrm{H}, J=8.8 \mathrm{~Hz}), 7.49-7.31$ $(\mathrm{m}, 8 \mathrm{H}), 6.01\left(\mathrm{~s}, 2 \mathrm{H}, \mathrm{CH}_{2}, \mathrm{H} 13\right), 1.56\left(\mathrm{~s}, 9 \mathrm{H}, \mathrm{CH}_{3}, \mathrm{H} 4\right), 1.49 \mathrm{ppm}(\mathrm{s}$, $\left.9 \mathrm{H}, \mathrm{CH}_{3}, \mathrm{H} 8\right) ;{ }^{13} \mathrm{C}$ NMR $\left(100 \mathrm{MHz}, \mathrm{CDCl}_{3}\right): \delta_{\mathrm{C}}=148.1,146.1,141.2$, $137.7,134.5,130.9,129.1,127.8,127.5,126.7,126.0,125.4,124.5$, 124.0, 123.8, 123.5, 122.7, 121.8, 121.1, 120.6, 119.7, 119.0, 50.1, 31.6, 31.4 ppm; ESMS: calc for $\mathrm{C}_{35} \mathrm{H}_{36} \mathrm{~N}\left([2.22+\mathrm{H}]^{+}\right)=470.2842$, found 470.2830, calc for $\mathrm{C}_{35} \mathrm{H}_{35} \mathrm{~N}\left([2.22 \mathrm{M}]^{+}\right)=469.2763$, found 469.2769; IR (KBr): $\tilde{v}=2962(\mathrm{~s}), 2903(\mathrm{~m}, \mathrm{sh}), 2868(\mathrm{~m}, \mathrm{sh}), 1568(\mathrm{~m})$, $1525(\mathrm{~m}), 1468(\mathrm{~s}), 1454(\mathrm{~s}), 1358(\mathrm{~s}), 1258(\mathrm{~m}), 948(\mathrm{~m}), 810(\mathrm{~s}), 733$ (s), $600 \mathrm{~cm}^{-1}(\mathrm{~m}) ;$ UV/Vis $\lambda_{\max }(\varepsilon): 276 \mathrm{~nm}(66433), 297 \mathrm{~nm}(19674)$, $325 \mathrm{~nm}$ (20986), $360 \mathrm{~nm}$ (6390), $379 \mathrm{~nm}$ (6227); Fluorometry $\lambda_{\max }$ : $389 \mathrm{~nm}, 407 \mathrm{~nm}$.

CCDC 1881745 (7a), 1881746 (7 d), 1881747 (7 h), 1881748 (10 c), $1881749(\mathbf{1 0} \mathrm{f})$, and $1881750(\mathbf{1 0} \mathbf{~ g})$ contain the supplementary crystallographic data for this paper. These data can be obtained free of charge from The Cambridge Crystallographic Data Centre.

\section{Supporting Information Available}

Included in the Electronic Supporting Information: - Experimental methods-synthesis of compound series 7 - Chemical structures for all molecules in our data set - Cartesian coordinates of ground state optimized reactant geometries for all molecules in our data set

\section{Conflict of interest}

The authors declare no conflict of interest.

Keywords: ab initio calculations - photocyclization photoplanarization • oxidative cyclodehydrogenation reactivity predictor

[1] H. D. Roth, Angew. Chem. Int. Ed. Engl. 1989, 28, 1193-1207; Angew. Chem. 1989, 101, 1220-1234.

[2] M. Oelgemöller, Chem. Rev. 2016, 116, 9664-9682.

[3] N. Hoffmann, Chem. Rev. 2008, 108, 1052-1103.

[4] R. Brimioulle, D. Lenhart, M. M. Maturi, T. Bach, Angew. Chem. Int. Ed. 2015, 54, 3872-3890; Angew. Chem. 2015, 127, 3944-3963.

[5] J. E. Anthony, Chem. Rev. 2006, 106, 5028-5048.

[6] C.-J. Zheng, W.-M. Zhao, Z.-Q. Wang, D. Huang, J. Ye, X.-M. Ou, X.-H. Zhang, C.-S. Lee, S.-T. Lee, J. Mater. Chem. 2010, 20, 1560-1566.

[7] P.-Y. Gu, Y. Zhao, J.-H. He, J. Zhang, C. Wang, Q.-F. Xu, J.-M. Lu, X. W. Sun, Q. Zhang, J. Org. Chem. 2015, 80, 3030-3035.

[8] Q. Zhang, Y. Divayana, J. Xiao, Z. Wang, E. Tiekink, H. Doung, H. Zhang F. Boey, X. Sun, F. Wudl, Chem. Eur. J. 2010, 16, 7422-7426. 
[9] Z. Zhao, S. Chen, J. W. Y. Lam, P. Lu, Y. Zhong, K. S. Wong, H. S. Kwok, B. Z. Tang, Chem. Commun. 2010, 46, 2221-2223.

[10] M. Vasilopoulou, L. C. Palilis, A. Botsialas, D. G. Georgiadou, P. Bayiati, N. Vourdas, P. S. Petrou, G. Pistolis, N. A. Stathopoulos, P. Argitis, Phys. Status Solidi 2008, 5, 3658-3662.

[11] T. Hasegawa, J. Takeya, Sci. Technol. Adv. Mater. 2009, 10, 024314.

[12] R. Jin, K. Wang, Int. J. Mol. Sci. 2015, 16, 20326-20343.

[13] M. Scharber, N. Sariciftci, Prog. Polym. Sci. 2013, 38, 1929-1940.

[14] L. Kazmerski in Comprehensive Renewable Energy (Ed.: A. Sayigh), Elsevier, Oxford, 2012, pp. 13-30.

[15] R. J. Cogdell, T. H. Brotosudarmo, A. T. Gardiner, P. M. Sanchez, L. Cronin Biofuels 2010, 1, 861-876.

[16] T. Moore, A. Moore, D. Gust, J. Barber, R. Van Grondelle, L. Sun, L. Hammarstrom, Philos. Trans. R. Soc. London Ser. B 2002, 357, 1481-1498.

[17] Z. Bao, Organic Materials for Thin Film Transistors, https://www.sigmaaldrich.com/technical-documents/articles/materialmatters/organic-materials.html, 2007.

[18] B. Kumar, B. K. Kaushik, Y. S. Negi, Polym. Rev. 2014, 54, 33-111.

[19] H. Klauk, Chem. Soc. Rev. 2010, 39, 2643-2666.

[20] L.-K. Mao, J.-C. Hwang, T.-H. Chang, C.-Y. Hsieh, L.-S. Tsai, Y.-L. Chueh, S. S. Hsu, P.-C. Lyu, T.-J. Liu, Org. Electron. 2013, 14, 1170-1176.

[21] C. Reese, M. Roberts, M. Ling, Z. Bao, Mater. Today 2004, 7, 20-27.

[22] Z. Wang, X. Niu, X. Zhou, R. Song, Z. Wang, L. Huang, L. Chi, Org. Electron. 2018, 58, 38-45.

[23] Y. Shibata, T. Matsuzaki, T. Ishinabe, H. Fujikake, J. Cryst. Growth 2018 492, 98- 104.

[24] Y. Takashima, K. Fukudome, A. Horikoshi, T. Tsuruoka, K. Akamatsu, Polyhedron 2018, 155, 275-280.

[25] W.-C. Wang, T.-T. Yeh, W.-L. Liau, J.-T. Chen, C.-S. Hsu, Org. Electron. 2018 $57,82-88$.

[26] F. Lefèvre, P. Juneau, R. Izquierdo, Sens. Actuators B 2015, 221, 1314 1320.

[27] M. Pięk, B. Paczosa-Bator, J. Smajdor, R. Piech, Electrochim. Acta 2018 283, 1753-1762.

[28] A. U. Alam, Y. Qin, S. Nambiar, J. T. Yeow, M. M. Howlader, N.-X. Hu, M. J. Deen, Prog. Mater. Sci. 2018, 96, 174-216.

[29] F. B. Mallory, C. S. Wood, J. T. Gordon, J. Am. Chem. Soc. 1964, 86, 3094 3102.

[30] A. D. D. Wonanke, D. L. Crittenden, Aust. J. Chem. 2018, 71, 249-256.

[31] F. B. Mallory, C. W. Mallory, Photocyclization of Stilbenes and Related Molecules, Wiley, 2004, pp. 1-456.

[32] F. B. Mallory, K. E. Butler, A. C. Evans, E. J. Brondyke, C. W. Mallory, C Yang, A. Ellenstein, J. Am. Chem. Soc. 1997, 119, 2119-2124.
[33] L. Liu, B. Yang, T. J. Katz, M. K. Poindexter, J. Org. Chem. 1991, 56, 3769 3775.

[34] M. S. Molloy, J. A. Snyder, A. E. Bragg, J. Phys. Chem. A 2014, 118, $3913-$ 3925.

[35] W. M. Moore, D. D. Morgan, F. R. Stermitz, J. Am. Chem. Soc. 1963, 85 $829-830$.

[36] W. H. Laarhoven, Recl. Trav. Chim. Pays-Bas 1983, 102, 185-204.

[37] W. Laarhoven, T. Cuppen, R. Nivard, Tetrahedron 1970, 26, 1069-1083.

[38] M. Scholz, M. Muhlstadt, F. Dietz, Tetrahedron Lett. 1967, 8, 665-668.

[39] K. A. Muszkat, G. Seger, S. Sharafi-Ozeri, J. Chem. Soc. Faraday Trans. 2 $1975,71,1529-1544$

[40] K. Muzkat, S. Sharafi-Ozeri, Chem. Phys. Lett. 1973, 20, 397-400.

[41] P. R.-G. C. Minot, C. Thal, Tetrahedron 1980, 36, 1209-1214.

[42] A. Streitwieser, Jr., Molecular orbital theory for organic chemists, Wiley, 1961.

[43] S. M. Bachrach, Rev. Comput. Chem. 1994, 171-228.

[44] P. H.-Y. Cheong, C. Y. Legault, J. M. Um, N. Celebi-Olcum, K. N. Houk, Chem. Rev. 2011, 111, 5042-5137.

[45] T. Sperger, I. A. Sanhueza, F. Schoenebeck, Acc. Chem. Res. 2016, 49, $1311-1319$.

[46] A. D. Becke, J. Chem. Phys. 1993, 98, 5648-5652.

[47] C. Lee, W. Yang, R. G. Parr, Phys. Rev. B 1988, 37, 785-789.

[48] P. J. Stephens, F. J. Devlin, C. F. Chabalowski, M. J. Frisch, J. Phys. Chem 1994, 98, 11623-11627.

[49] H. Kruse, L. Goerigk, S. Grimme, J. Org. Chem. 2012, 77, 10824-10834.

[50] F. B. Mallory, C. W. Mallory, Photocyclization of Stilbenes and Related Molecules, American Cancer Society, 2005, chap. 1, pp. 1-456.

[51] E. Fischer, J. Larsen, J. B. Christensen, M. Fourmigue, H. G. Madsen, N. Harrit, J. Org. Chem. 1996, 61, 6997-7005.

[52] M. Ogata, H. Matsumoto, Chem. Pharm. Bull. 1972, 20, 2264-2268.

[53] W. H. Laarhoven, T. J. R. M. Cuppen, R. J. F. Nivard, Recl. Trav. Chim. PaysBas 1968, 87, 686-698.

[54] J. L. Ferguson, M. A. Squire, C. M. Fitchett, Org. Biomol. Chem. 2017, 15, 9293-9296.

[55] T. Yanai, D. P. Tew, N. C. Handy, Chem. Phys. Lett. 2004, 393, 51-57.

[56] M. K. Węcławski, M. Tasior, T. Hammann, P. J. Cywinski, D. T. Gryko, Chem. Commun. 2014, 50, 9105-9108.

Manuscript received: November 30, 2018

Revised manuscript received: February 1, 2019

Accepted manuscript online: February 4, 2019

Version of record online: February 20, 2019 